\title{
Diabetic ketoacidosis and hyperosmolar hyperglycemic syndrome: review of acute decompensated diabetes in adult patients
}

\author{
Esra Karslioglu French, ${ }^{1}$ Amy C Donihi, ${ }^{2}$ Mary T Korytkowski ${ }^{1}$
}

${ }^{1}$ Division of Endocrinology and Metabolism, Department of Medicine, University of Pittsburgh, Pittsburgh, PA, USA

${ }^{2}$ University of Pittsburgh School of Pharmacy, Pittsburgh, PA, USA Correspondence to: M Korytkowski mtk7@pitt.edu

Cite this as: BMJ 2019;365:11114 doi: 10.1136/bmj.l1114

Series explanation: State of the Art Reviews are commissioned on the basis of their relevance to academics and specialists in the US and internationally. For this reason they are written predominantly by US authors

\begin{abstract}
Diabetic ketoacidosis and hyperosmolar hyperglycemic syndrome (HHS) are life threatening complications that occur in patients with diabetes. In addition to timely identification of the precipitating cause, the first step in acute management of these disorders includes aggressive administration of intravenous fluids with appropriate replacement of electrolytes (primarily potassium). In patients with diabetic ketoacidosis, this is always followed by administration of insulin, usually via an intravenous insulin infusion that is continued until resolution of ketonemia, but potentially via the subcutaneous route in mild cases. Careful monitoring by experienced physicians is needed during treatment for diabetic ketoacidosis and HHS. Common pitfalls in management include premature termination of intravenous insulin therapy and insufficient timing or dosing of subcutaneous insulin before discontinuation of intravenous insulin. This review covers recommendations for acute management of diabetic ketoacidosis and HHS, the complications associated with these disorders, and methods for preventing recurrence. It also discusses why many patients who present with these disorders are at high risk for hospital readmissions, early morbidity, and mortality well beyond the acute presentation.
\end{abstract}

\section{Introduction}

Diabetic ketoacidosis and hyperosmolar hyperglycemic syndrome (HHS) are life threatening emergencies that occur in patients with type 1 diabetes and type 2 diabetes. ${ }^{1-4}$ Diabetic ketoacidosis is defined by a triad of hyperglycemia (or a diagnosis of diabetes), metabolic acidosis, and ketonemia (table 1). ${ }^{12-7}$ HHS is defined by severe hyperglycemia, high serum osmolality, and dehydration (table 2). ${ }^{48}$ The presentation of each of these diabetic emergencies often overlaps. ${ }^{38}$ Early diagnosis and management with attention to prevention strategies are essential to improve patients' outcomes. ${ }^{12}$ This is particularly important for patients presenting with "euglycemic" diabetic ketoacidosis, which is a term used to describe diabetic ketoacidosis accompanied by lower than anticipated blood glucose. ${ }^{157910}$

The target audience for this review is endocrinologists, hospital based physicians, and other physicians or healthcare providers involved in the care of adult patients with or at risk for diabetic ketoacidosis and HHS. We present the epidemiology, pathophysiology, precipitating causes, clinical presentation, acute management, complications, and prevention of these disorders. In addition, we review some of the controversies and observed pitfalls in the management of these patients.

\section{Sources and section criteria}

We searched PubMed from May 1975 through January 2019 to identify English language articles published in peer reviewed journals by using the following search terms: diabetic ketoacidosis, euglycemic diabetic ketoacidosis, hyperosmolar hyperglycemic syndrome, hyperglycemic crisis, sodium glucose cotransporter 2 (SGLT2) inhibitors, protocols, cerebral edema, and hyperchloremic metabolic acidosis. We also identified references from relevant review articles, as well as from the similar items section of PubMed. We screened and reviewed more than 200 articles in the preparation of this manuscript. We reviewed only articles including humans. We considered inclusion of studies with smaller numbers of participants to be important, as these make up the majority of prospective studies for both diabetic ketoacidosis and HHS. Retrospective database reviews are also included, as is one case report illustrating the rare development of cerebral edema in an adult patient. ${ }^{11}$ We excluded articles published in non-peer reviewed journals or those that were not available in PubMed. We prioritized publications within the past decade covering the epidemiology and treatment of diabetic ketoacidosis and HHS in adult patients. We also prioritized inclusion of manuscripts that provided elegant examinations of the pathophysiology of 


\begin{tabular}{|c|c|c|c|}
\hline Criteria & $\mathrm{ADA}^{1}$ & $\mathrm{UK}^{2}$ & $\mathrm{AACE} / \mathrm{ACE}^{5}$ \\
\hline Year of publication & 2009 & 2013 & 2016 \\
\hline $\begin{array}{l}\text { Plasma glucose concentration, } \\
\mathrm{mmol} / \mathrm{L}\end{array}$ & $>13.9(250 \mathrm{mg} / \mathrm{dL})^{\star}$ & $\begin{array}{l}>11 \text { (>200 mg/dL)or } \\
\text { known diabetes }\end{array}$ & NA \\
\hline $\mathrm{pH}$ & $\begin{array}{l}\text { Mild: } 7.25-7.30 \text {; moderate: } \\
7.00-7.24 ; \text { severe: }<7.00\end{array}$ & <7.3 (severe: «7.0) & $<7.3$ \\
\hline $\begin{array}{l}\text { Bicarbonate concentration, } \\
\mathrm{mmol} / \mathrm{L} \text { or } \mathrm{mEq} / \mathrm{L}\end{array}$ & $\begin{array}{l}\text { Mild: } 15-18 ; \text { moderate: } \\
\text { 10-14.9; severe: }<10\end{array}$ & «15 (severe: «5) & NA \\
\hline Anion gap: $\mathrm{Na}^{+}-\left(\mathrm{Cl}^{-}+\mathrm{HCO}_{3}^{-}\right)$ & $\begin{array}{l}\text { Mild: }>10 ; \text { moderate: }>12 \text {; } \\
\text { severe: }>12\end{array}$ & NA (severe: >16) & $>10$ \\
\hline $\begin{array}{l}\text { Urine acetoacetate } \\
\text { (nitroprusside reaction) }\end{array}$ & Positive & Positive & Positive \\
\hline $\begin{array}{l}\text { Blood } \beta \text {-hydroxybutyrate, } \\
\mathrm{mmol} / \mathrm{L}\end{array}$ & $N A^{\dagger}$ & 23 (31 mg/dL) (severe: >6) & $\geq 3.8(40 \mathrm{mg} / \mathrm{dL})$ \\
\hline Mental status & $\begin{array}{l}\text { Mild: alert; moderate: alert or } \\
\text { drowsy; severe: stupor or coma }\end{array}$ & NA & $\begin{array}{l}\text { Drowsy, stupor, or } \\
\text { coma }\end{array}$ \\
\hline \multicolumn{4}{|c|}{$\begin{array}{l}\text { AACE/ACE=American Association of Clinical Endocrinologists/American College of Endocrinology; ADA=American Diabetes } \\
\text { Association; NA=not included in guideline document. } \\
\text { *2019 ADA guideline provides update to this } 2009 \text { guideline and now states "There is considerable variability... ranging from } \\
\text { euglycemia or mild hyperglycemia and acidosis to severe hyperglycemia, dehydration, and coma." } \\
\text { † } \beta \text {-hydroxybutyrate updated to }>3 \text { mmol/L in } 2016 \text { updated review cited in } 2019 \text { ADA guideline. }{ }^{67}\end{array}$} \\
\hline
\end{tabular}

\begin{tabular}{|c|c|c|}
\hline Criteria & $\mathrm{ADA}^{1}$ & $\mathrm{UK}^{4}$ \\
\hline Year of publication & 2009 & 2015 \\
\hline Plasma glucose concentration, $\mathrm{mmol} / \mathrm{L}$ & 133.3 & $\geq 30$ \\
\hline $\mathrm{pH}$ & $>7.30$ & $>7.30$ \\
\hline Bicarbonate concentration, $\mathrm{mmol} / \mathrm{L}$ & $>18^{*}$ & $>15$ \\
\hline Anion gap: $\mathrm{Na}^{+}-\left(\mathrm{Cl}^{-}+\mathrm{HCO}_{3}^{-}\right)$ & NA & NA \\
\hline Urine acetoacetate (nitroprusside reaction) & Negative or low positive & NA \\
\hline Blood $\beta$-hydroxybutyrate, $\mathrm{mmol} / \mathrm{L}$ & NA & $<3$ \\
\hline Osmolality, $\mathrm{mmol} / \mathrm{kg}$ & $>320^{+}$ & $\geq 320^{\ddagger}$ \\
\hline Presentation & Stupor or coma & Severe dehydration and feeling unwell \\
\hline \multicolumn{3}{|c|}{$\begin{array}{l}\text { ADA=American Diabetes Association; NA=not included in guideline document. } \\
\text { *Updated to }>15 \mathrm{mmol} / \mathrm{L} \text { in } 2016 \text { updated review cited in } 2019 \text { ADA guideline }{ }^{67} \\
\text { tADA guideline calculates effective plasma osmolality using equation } 2 \times \mathrm{Na}+\text { glucose }(\mathrm{mmol} / \mathrm{L}) \text { or } 2 \times \mathrm{Na}+\text { glucose }(\mathrm{mg} / \\
\mathrm{dL}) / 18 \text {. } \\
\text { fUK guideline calculates osmolality using equation } 2 \times \mathrm{Na}+\text { glucose }(\mathrm{mmol} / \mathrm{L})+(\text { blood urea nitrogen }(\mathrm{mmol} / \mathrm{L}) \text { or } \\
2 \times \mathrm{Na}+\text { glucose }(\mathrm{mg} / \mathrm{dL}) / 18+\text { blood urea nitrogen }(\mathrm{mg} / \mathrm{dL}) / 2.8\end{array}$} \\
\hline
\end{tabular}

both diabetic ketoacidosis and HHS that were published between 1980 and 1987, as it is unlikely that these studies will be repeated. We included randomized controlled trials (RCTs) and original articles published in the past 40 years, as well as consensus statements, guidelines, and systematic reviews published in the past 10 years. A supplementary table summarizing the objectives and key findings from several studies of the epidemiology, treatment strategies, and outcomes is available.

\section{Epidemiology}

The frequency of diabetic ketoacidosis has increased during the past decade, with more than 160000 hospital admissions in 2017 in the US. ${ }^{1213}$ A recent analysis found that hospital admissions for diabetic ketoacidosis had increased in the UK for patients with both type 1 and type 2 diabetes. ${ }^{14} \mathrm{HHS}$ is less common and accounts for less than $1 \%$ of all diabetes related admissions. ${ }^{15}$

The observed increase in hospital admissions for diabetic emergencies has several potential explanations. These include the rising prevalence of diabetes, as well as psychosocial, cultural, and economic factors that often limit access to insulin and outpatient medical care. ${ }^{131617}$ Diabetic ketoacidosis causes an estimated medical expend- iture of $\$ 2.4 \mathrm{bn}$ ( $€ 1.9 \mathrm{bn}$; $€ 2.1 \mathrm{bn}$ ) per year in the $\mathrm{US} .{ }^{10}$ In the UK, the average cost for an episode of diabetic ketoacidosis is estimated to be $€ 2064$ ( $\$ 2682$; €2384) per patient. ${ }^{18}$

Diabetic ketoacidosis was a fatal disease before the discovery of insulin in 1921. Nearing 100 years of insulin availability, mortality related to diabetic ketoacidosis in the adult population has progressively declined to less than $1 \%{ }^{13}$ Certain groups of patients have mortality rates of up to $5 \%$, including patients with significant comorbid conditions and those of advanced age. ${ }^{19} \mathrm{HHS}$, which occurs more commonly in older adults with underlying comorbidities, has a $10-20 \%$ mortality rate. ${ }^{820}$

Interest has been renewed in euglyemic diabetic ketoacidosis, which was first described as a case series of 37 patients presenting with blood glucose below 300 $\mathrm{mg} / \mathrm{dL} .{ }^{9}$ Euglycemic diabetic ketoacidosis can result in delayed diagnosis and treatment, as well as potential for adverse metabolic consequences. ${ }^{17}$ Since the recent introduction of the SGLT2 inhibitors, several case reports and series describing euglycemic diabetic ketoacidosis in patients treated with these agents have been published. ${ }^{21-23}$ The entity of euglycemic diabetic ketoacidosis is not limited to patients using SGLT2 inhibitors, however, as it has also been described in the setting of alcohol use disorders, pregnancy, and chronic liver disease..$^{924}$

\section{Pathophysiology}

Both diabetic ketoacidosis and HHS result from relative or absolute insulin deficiency together with an increase in circulating concentrations of counterregulatory hormones (figure). ${ }^{125}$

\section{Pathophysiology of diabetic ketoacidosis}

In the usual clinical situation, a rise in counterregulatory hormones contributes to accelerated gluconeogenesis, glycogenolysis, and impaired glucose utilization by peripheral tissues and leads to diabetic ketoacidosis. ${ }^{25}$ Glucagon is the primary counterregulatory hormone responsible for development of diabetic ketoacidosis, as increases in other counterregulatory hormones (catecholamines, cortisol, and growth hormone) are not necessarily observed. ${ }^{1}$ However, even glucagon is not absolutely essential, as diabetic ketoacidosis has also been described in patients after pancreatectomy. ${ }^{25} 26$

In the liver, the gluconeogenic enzymes fructose 1,6 bisphosphatase, phosphoenolpyruvate carboxykinase (PEPCK), glucose-6-phosphatase, and pyruvate carboxylase are stimulated by an increase in the glucagon to insulin ratio and by an increase in circulating cortisol concentrations. ${ }^{27}{ }^{28}$ Hepatic gluconeogenesis is the main mechanism for hyperglycemia in ketoacidosis, but renal gluconeogenesis also contributes..$^{29-31}$

In adipose tissue, the combination of severe insulin deficiency with elevated counterregulatory hormone concentrations activates hormone sensitive lipase leading to an increase in circulating free fatty acids. ${ }^{31-34}$ Excess free fatty acids are oxidized to acetoacetate and $\beta$-hydroxybutyrate in hepatic mitochondria, resulting in ketonemia and acidosis. ${ }^{35}$ Glucagon accelerates the generation of ketonemia and hyperglycemia in the insulin deficient state, but as previously mentioned it is 

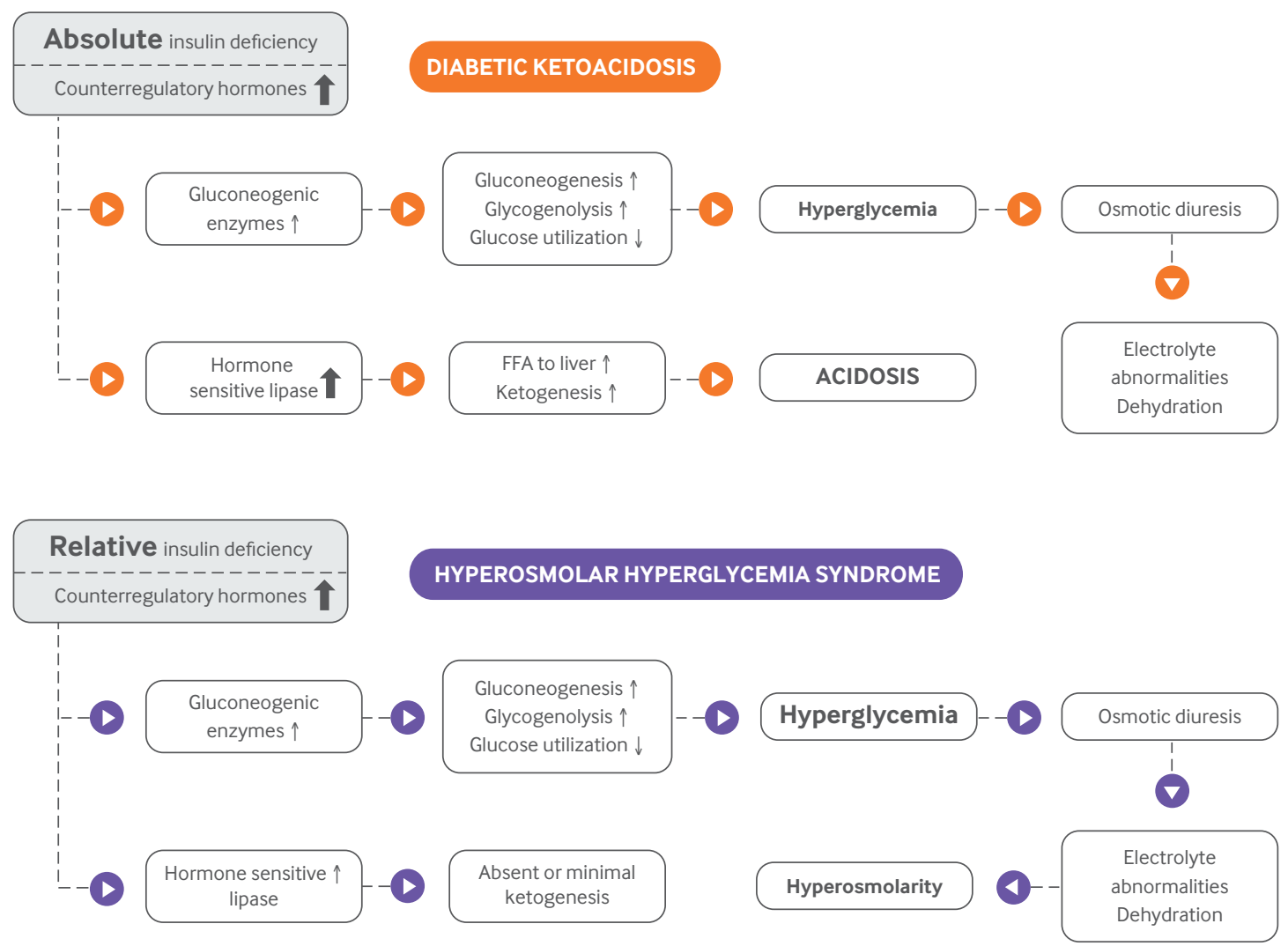

Pathogenesis of diabetic ketoacidosis and hyperosmolar hyperglycemic syndrome. FFA=free fatty acids

not essential for development of diabetic ketoacidosis. In addition to ketone body overproduction, clearance is also decreased in diabetic ketoacidosis. ${ }^{25} 36$

Both hyperglycemia and high circulating concentrations of ketone bodies result in an osmotic diuresis, which leads to hypovolemia and subsequent decrease in glomerular filtration rate. Osmotic diuresis promotes net loss of sodium, potassium, calcium, magnesium, chloride, and phosphate, further contributing to electrolyte abnormalities associated with diabetic ketoacidosis. ${ }^{25}$ Progressive volume depletion leads to decreased glomerular filtration rate, with further decreases in clearance of glucose and ketone bodies further contributing to hyperglycemia, hyperosmolality, and metabolic acidosis. ${ }^{25}$

\section{Pathophysiology of HHS}

The pathogenesis of HHS differs from that of diabetic ketoacidosis in that a more severe degree of dehydration is present owing to osmotic diuresis and an absence of significant ketosis/ketonemia. ${ }^{1347}$ A higher concentration of circulating hepatic insulin could partly account for the absence of significant ketosis in patients with HHS. ${ }^{3}$ Patients presenting with HHS have lower concentrations of free fatty acids, cortisol, growth hormone, and glucagon than do those presenting with diabetic ketoacidosis. Patients with HHS may have mild metabolic acidosis due to renal failure and dehydration. ${ }^{20}$

\section{Precipitating causes}

The most common precipitating factors in diabetic ketoacidosis and HHS are inadequate insulin therapy and infection, followed by new onset of diabetes and other metabolic stressors. ${ }^{37-39}$ Certain drugs including glucocorticoids, excess diuretics, atypical antipsychotics, and others can predispose to severe hyperglycemia, diabetic ketoacidosis, and HHS. ${ }^{2040}$

\section{Precipitating causes of diabetic ketoacidosis}

In a large survey of 283 patients admitted to one of 72 hospitals in the UK, infection was identified as the most common precipitating factor for diabetic ketoacidosis (45\%), followed by insulin omission (20\%); other causes included newly diagnosed diabetes and alcohol or drug related problems..$^{41}{ }^{42}$ Insulin omission can be seen in patients of all ages and is more commonly observed in those with eating disorders, psychological distress, fear of hypoglycemia, and fear of weight gain. ${ }^{39}$ Other factors that are associated with insulin omission include inability to pay for insulin, the idea that insulin should be withheld when illness interferes with eating, inadvertent omission of an insulin dose, and, rarely, pump malfunction. ${ }^{173839}$

It is not only patients who stop their insulin therapy. In the UK survey described above, more than $7 \%$ of cases of diabetic ketoacidosis occurred in an inpatient population. ${ }^{41}$ We (and patients who reviewed this manuscript) have observed situations in which insulin therapy is erroneously withheld in patients admitted to hospital with type 1 diabetes who are placed in a fasting state for surgical or other procedures. Some healthcare providers also make erroneous assumptions that patients over 50 years of age have type 2 diabetes and can tolerate periods 
of insulin omission when admitted to hospital for acute illness or surgical procedures (based on personal observations and experiences). Additionally, insulin therapy can be erroneously withheld or discontinued in patients admitted to the hospital with an insulin pump device owing to a lack of familiarity with these devices on the part of hospital personnel. . $^{43-45}$

SGLT2 inhibitors have been identified as causal agents in several reported cases of euglycemic diabetic ketoacidosis. ${ }^{23}{ }^{46} \mathrm{~A}$ discussion of how these agents contribute to diabetic ketoacidosis is beyond the scope of this manuscript, and readers are referred to several publications in which this is discussed..$^{47-49}$ Risk factors for euglycemic diabetic ketoacidosis with SGLT2 inhibitors include latent autoimmune diabetes of adulthood, surgery, low carbohydrate diets, insulin withdrawal or dose reduction, and acute medical illness.

Other clinical scenarios associated with euglycemic diabetic ketoacidosis include pregnancy, decreased caloric intake, heavy alcohol use, and chronic liver disease. ${ }^{2150}$ An awareness of these factors is important so as to avoid missing a diagnosis of diabetic ketoacidosis and delaying treatment. ${ }^{50}$

\section{Precipitating causes of HHS}

HHS occurs most commonly, but not exclusively, in older people with type 2 diabetes and accompanying comorbidities. Precipitating factors include pneumonia (40$60 \%$ ) and urinary tract infections (5-16\%) or other acute conditions such as cerebrovascular disease, myocardial infarction, or trauma. ${ }^{32051}$ The risk of HHS increases in settings of inadequate fluid intake due to altered thirst mechanisms with aging or inability to access fluids. ${ }^{51}$

\section{Clinical presentation and diagnosis}

Table 1 and table 2 outline diagnostic criteria for diabetic ketoacidosis and HHS as recommended by the American Diabetes Association (ADA), Joint British Diabetes Societies for Inpatient Care, and American Association of Clinical Endocrinologists. Diagnostic criteria for diabetic ketoacidosis can include an elevation of urine acetoacetate or blood $\mathrm{D-hydroxybutyrate.} \mathrm{Point} \mathrm{of} \mathrm{care}$ blood ketone meters and test strips for measurement of प-hydroxybutyrate are costly and not readily available in many institutions, but they are likely to become standard of care over time as they also provide accurate information for guiding treatment (see management section below). ${ }^{36}$

Patients with diabetic ketoacidosis can present with some or all of the following symptoms: polyuria, polydipsia, nausea, vomiting, abdominal pain, visual disturbance, lethargy, altered sensorium, tachycardia, tachypnea, and Kussmaul respirations, with a fruity odor to the breath. Patients are usually severely volume depleted with orthostatic hypotension. Patients with euglycemic diabetic ketoacidosis secondary to treatment with a SGLT2 inhibitor may have less polyuria and polydipsia owing to the milder degree of hyperglycemia and may instead present with malaise, anorexia, tachycardia, or tachypnea with or without fever. ${ }^{24}$ Patients with HHS often present with an altered level of consciousness, which may mask the usual symptoms of hyperglycemia.
A direct relation exists between hyperosmolality and depressed sensorium in patients presenting with blood glucose above $33.3 \mathrm{mmol} / \mathrm{L}(600 \mathrm{mg} / \mathrm{dL})$ and insignificant concentrations of ketones. ${ }^{2052}$ Patients presenting with diabetic ketoacidosis, including euglycemic diabetic ketoacidosis, and HHS need immediate referral for emergency evaluation and treatment.

\section{Acute management}

The management of both diabetic ketoacidosis and HHS includes fluids (usually administered intravenously), electrolytes, and insulin. Identifying the cause of acute decompensated diabetes is important, but this should not cause any delay in treatment. Patients (and their family members), especially those who present with more severe symptoms of diabetic ketoacidosis or HHS, often describe anxiety as part of their acute presentation. An explanation of what is happening, and the anticipated course of management, can alleviate some of these concerns and open discussions as to how this can be prevented in the future (see prevention section below).

Use of standardized paper based and computerized guidelines and protocols for management of diabetic ketoacidosis has been shown to decrease the time to anion gap closure, reduce length of stay in hospital, and minimize complications during treatment for diabetic ketoacidosis. ${ }^{53-57}$ However, even when protocols are available and healthcare providers are aware of them, adherence is often poor. ${ }^{414258}$ Treatment protocols must be designed to be flexible and simple to use by physicians, nurses, and other healthcare providers, as the management of diabetic ketoacidosis and HHS is complicated, requiring close monitoring and modification over the duration of treatment. It is especially important to recognize that guidelines and order sets that are written or embedded in an electronic health record do not substitute for sound clinical judgment. ${ }^{59}$

\section{Management of diabetic ketoacidosis}

The goals of management of diabetic ketoacidosis include restoration of intravascular volume, prevention and/or correction of electrolyte abnormalities, correction of acidosis, and correction of hyperglycemia (if present) (box 1). ${ }^{1763}$ Patients presenting with mild diabetic ketoacidosis who are alert and able to tolerate oral fluids may be able to receive treatment in the emergency department, potentially with oral fluids and subcutaneous insulin, and without need for hospital admission. ${ }^{64}$ Patients presenting with more severe degrees of metabolic derangement need to be admitted to a hospital unit with trained personnel and resources for intensive monitoring and administration of intravenous fluids, potassium, and insulin. The ADA and the Joint British Diabetes Societies for Inpatient Care have both published guidelines for management of diabetic ketoacidosis, but these guidelines have several differences. These differences are primarily due to lack of published evidence to guide treatment in many areas. ${ }^{63}$ Despite this, the ADA guideline has been widely distributed, is used internationally, and has been cited more than 600 times..$^{63}$ In addition, a survey of 118 UK clinical diabetes teams found that the UK guideline has been adopted by more than $90 \%$ of those surveyed. ${ }^{1065}$ 
Box 1 Principles of diabetic ketoacidosis (DKA) management in adult patients

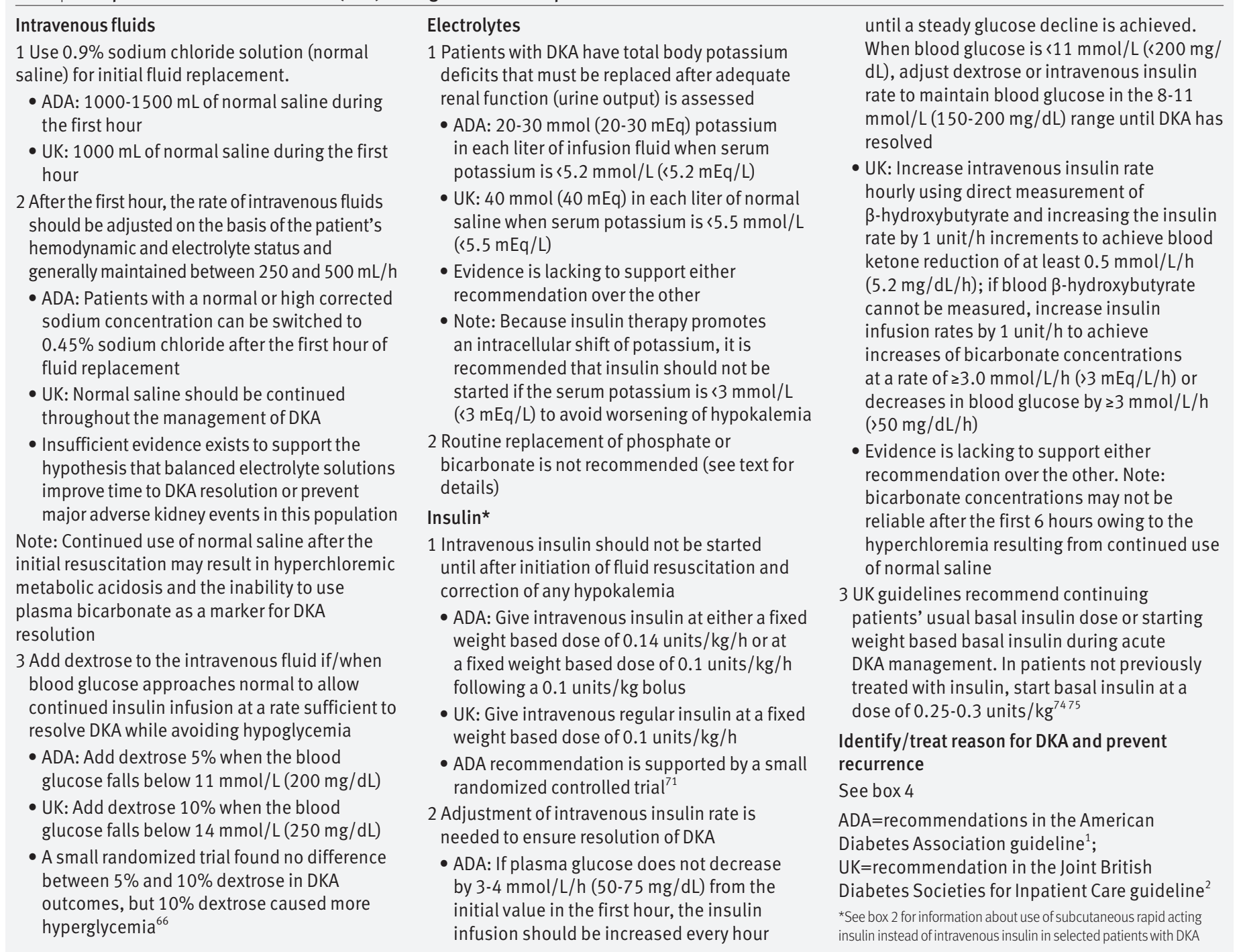

\section{Intravenous fluids}

Administration of fluid is the first line of treatment. Appropriate fluid administration not only restores intravascular volume but also lowers blood glucose, raises blood pressure, ensures perfusion of peripheral tissues, and facilitates resolution of metabolic acidosis. Current ADA and UK guidelines for management of diabetic ketoacidosis in adults both recommend $0.9 \%$ sodium chloride solution (normal saline) for initial fluid replacement. ${ }^{12}$ Specifically, the ADA recommends $1000-1500 \mathrm{~mL}$ and the UK guideline recommends $1000 \mathrm{~mL}$ of normal saline during the first hour. Although normal saline contains a supraphysiologic concentration of chloride potentially leading to hyperchloremic metabolic acidosis, as discussed in the complications section of this review, insufficient evidence exists to support the hypothesis that balanced electrolyte solutions improve time to resolution of diabetic ketoacidosis or prevent major adverse kidney events in this population. ${ }^{60}$ The UK guideline acknowledges this and includes a statement that balanced electrolyte solutions can be used, but as they are not commercially available with premixed potassium chloride, they must be used in conjunction with additional potassium replacement. $^{2}$
After the initial hour, the rate of intravenous fluids should be adjusted on the basis of the patient's hemodynamic and electrolyte status and generally maintained between 250 and $500 \mathrm{~mL} / \mathrm{h}$ in adult patients without cardiac or renal compromise, advanced liver disease, or other states of fluid overload. No RCTs are available to guide the appropriate rate of intravenous fluids. The ADA guideline recommends that patients with a normal or high corrected sodium concentration can be switched to $0.45 \%$ sodium chloride after the first hour of fluid replacement. ${ }^{1}$ However, the UK guideline recommends the continuation of normal saline throughout the management of diabetic ketoacidosis. ${ }^{2}$ No published studies support or refute a switch to $0.45 \%$ sodium chloride; however, continued use of normal saline after the initial resuscitation may result in hyperchloremic metabolic acidosis and the inability to use plasma bicarbonate as a marker for resolution of diabetic ketoacidosis.

As glucose normalizes with treatment, dextrose must be added to the intravenous fluid to allow continued insulin infusion at a rate sufficient to resolve ketonemia while avoiding hypoglycemia. The UK guideline recommends adding dextrose $10 \%$ when the blood glucose falls below $13.9 \mathrm{mmol} / \mathrm{L}(250 \mathrm{mg} / \mathrm{dL}){ }^{2}$ The ADA recommends adding 
dextrose $5 \%$ when the blood glucose falls below $11 \mathrm{mmol} / \mathrm{L}$ (200 mg/dL). ${ }^{1}$ A small randomized trial of 17 patients found no difference in capillary blood $\mathrm{pH}$ or bicarbonate concentration when $5 \%$ and $10 \%$ dextrose were compared, although $10 \%$ dextrose caused more hyperglycemia, with a mean blood glucose of $15.7 \mathrm{mmol} / \mathrm{L}$ at six hours compared with $11.5 \mathrm{mmol} / \mathrm{L}$ in the group receiving 5\% dextrose despite concurrent use of more insulin $(\mathrm{P}<0.05) .{ }^{66}$ One example diabetic ketoacidosis protocol instructs nurses to add 5\% dextrose at the same rate using the same concentrations of sodium chloride and potassium when blood glucose falls below $13.9 \mathrm{mmol} / \mathrm{L}(250 \mathrm{mg} / \mathrm{dL}){ }^{67}$ For example, $0.45 \%$ sodium chloride with $20 \mathrm{mEq}$ potassium chloride $(\mathrm{KCl})$ in each liter would change to dextrose $5 \%$ with $0.45 \%$ sodium chloride and $20 \mathrm{mEqKCl} / \mathrm{L}$ at the same infusion rate.

Another strategy that has recently emerged is use of a "two bag method" for fluid replacement. ${ }^{6869}$ This method consists of two bags of $0.45 \%$ sodium chloride, one with and one without $10 \%$ dextrose, that are adjusted on the basis of hourly blood glucose monitoring to maintain an intravenous fluid rate of $250 \mathrm{~mL} / \mathrm{h}$. Two retrospective studies involving more than 500 patients found that the two bag method was associated with earlier correction of acidosis and shorter duration of intravenous insulin compared with conventional delivery of intravenous fluids. ${ }^{6869}$ When used in the emergency department, this method may reduce the need for hospital admission, and it may be associated with less hypoglycemia compared with conventional treatment.

Of note, vulnerabilities in the intravenous fluid supply in the US have existed for several years, prompting some hospitals to develop protocols for oral rehydration in patients with dehydration. ${ }^{6170}$ Although these protocols have not been specifically tested in patients with diabetic ketoacidosis, this strategy can be considered in selected patients with mild-moderate diabetic ketoacidosis when accompanied by appropriate monitoring for therapeutic efficacy. ${ }^{61}$

\section{Potassium replacement}

Patients with diabetic ketoacidosis have total body potassium deficit despite measured serum potassium concentrations that may be normal or even high at presentation. The ADA recommends adding 20-30 mEq potassium in each liter of infusion fluid when serum potassium is below $5.2 \mathrm{mEq} / \mathrm{L}$. ${ }^{1}$ By contrast, the UK guideline recommends $40 \mathrm{mmol} / \mathrm{L}$ in each liter of normal saline when serum potassium is below $5.5 \mathrm{mmol} / \mathrm{L}$ and the patient is passing urine. ${ }^{2}$ As existing published trials evaluating potassium replacement rates were not designed to determine optimal potassium replacement rates in patients with diabetic ketoacidosis, evidence is lacking to support either recommendation over the other. ${ }^{60}$ Because insulin therapy promotes an intracellular shift of potassium, it is recommended that insulin should not be started if the serum potassium is below $3 \mathrm{mmol} / \mathrm{L}$ to avoid worsening of hypokalemia.

\section{Intravenous insulin}

The primary purpose of insulin in diabetic ketoacidosis management is to halt lipolysis and ketogenesis. Titration protocols for intravenous insulin infusion that are not designed for diabetic ketoacidosis management should not be used. Use of protocols that target pre-specified goal blood glucose ranges such as 6.1-7.8 mmol/L (110-140 $\mathrm{mg} / \mathrm{dL}$ ) or $7.8-10 \mathrm{mmol} / \mathrm{L}(140-180 \mathrm{mg} / \mathrm{dL})$ for patients in critical care settings can lead to inappropriately low doses or even premature discontinuation of intravenous insulin, which delays resolution of diabetic ketoacidosis. ${ }^{67}$ Even patients with euglycemic diabetic ketoacidosis need adequate insulin therapy to resolve ketonemia, albeit with early addition of fluids containing dextrose to prevent hypoglycemia. ${ }^{24}$

Insulin should not be started until after initiation of fluid resuscitation and correction of any hypokalemia to avoid worsening volume or potassium deficits with shifting of potassium, glucose, and water from the extracellular to intracellular fluids compartment. The UK guideline recommends starting intravenous regular insulin at a fixed weight based dose of 0.1 units $/ \mathrm{kg} / \mathrm{h} .{ }^{2}$ The ADA guideline recommends starting intravenous regular insulin at either a fixed weight based dose of 0.14 units $/ \mathrm{kg} / \mathrm{h}$ or a fixed weight based dose of 0.1 units $/ \mathrm{kg} / \mathrm{h}$ after a 0.1 units $/ \mathrm{kg}$ bolus of intravenous insulin. ${ }^{1}$ The ADA recommendation is based on a small RCT of 37 patients randomized to receive a loading dose of $0.07 \mathrm{unit} / \mathrm{kg}$ of regular insulin followed by $0.07 \mathrm{unit} / \mathrm{kg} / \mathrm{h}$, regular insulin 0.07 unit $/ \mathrm{kg} / \mathrm{h}$ without a loading dose, or regular insulin 0.14 unit $/ \mathrm{kg} / \mathrm{h}$ without a loading dose. ${ }^{71}$ Most patients in the $0.07 \mathrm{unit} / \mathrm{kg} / \mathrm{h}$ group without the loading dose needed supplemental doses of insulin to obtain desired changes in glucose; with these supplemental doses, no difference was seen in the time to resolution of diabetic ketoacidosis between the three groups. ${ }^{71}$ Although a rate of 0.1 unit/ $\mathrm{kg} / \mathrm{h}$ may not be sufficient to suppress hepatic glucose production and stimulate peripheral glucose uptake, it is likely sufficient to suppress lipolysis and ketogenesis. ${ }^{72}$

The ADA guideline states that reducing insulin infusion rates to $0.02-0.05$ units $/ \mathrm{kg} / \mathrm{h}$ at the same time that dextrose $5 \%$ is added to the intravenous fluids may be possible when blood glucose declines to below $11 \mathrm{mmol} / \mathrm{L}$ (200 mg/dL). ${ }^{1}$ However, this rate has not been proven to be sufficient to suppress lipolysis and ketosis. ${ }^{72}$ Additional studies are needed to assess whether the potential benefits of reduction of the insulin rate (reduced hypoglycemia and hypokalemia) justify the potential delay in resolution of diabetic ketoacidosis. The UK guideline does not recommend any adjustment in the insulin rate when dextrose $10 \%$ is added after the blood glucose falls below $14 \mathrm{mmol} / \mathrm{L}(252 \mathrm{mg} / \mathrm{dL}){ }^{265}$

After insulin has been started, the ADA recommends increasing insulin infusion rates hourly to reduce blood glucose at a rate of $3-4 \mathrm{mmol} / \mathrm{L} / \mathrm{h}(50-75 \mathrm{mg} / \mathrm{dL} / \mathrm{h})$ until concentrations of $8-11 \mathrm{mmol} / \mathrm{L}(150-200 \mathrm{mg} / \mathrm{dL})$ are achieved. ${ }^{1}$ On the other hand, the more recent UK guideline recommends using direct measurement of ๑-hydroxybutyrate (a hydroxy acid) and increasing the insulin rate by 1 unit/h increments to achieve blood ketone reduction of at least $0.5 \mathrm{mmol} / \mathrm{L} / \mathrm{h}(5.2 \mathrm{mg} / \mathrm{dL} / \mathrm{h}){ }^{2}$ The rate of glucose lowering is not directly correlated with the degree of ketonemia suppression and reversal of acidosis; however, point of care blood ketone meters are not available in all hospitals, and in those hospitals that do 


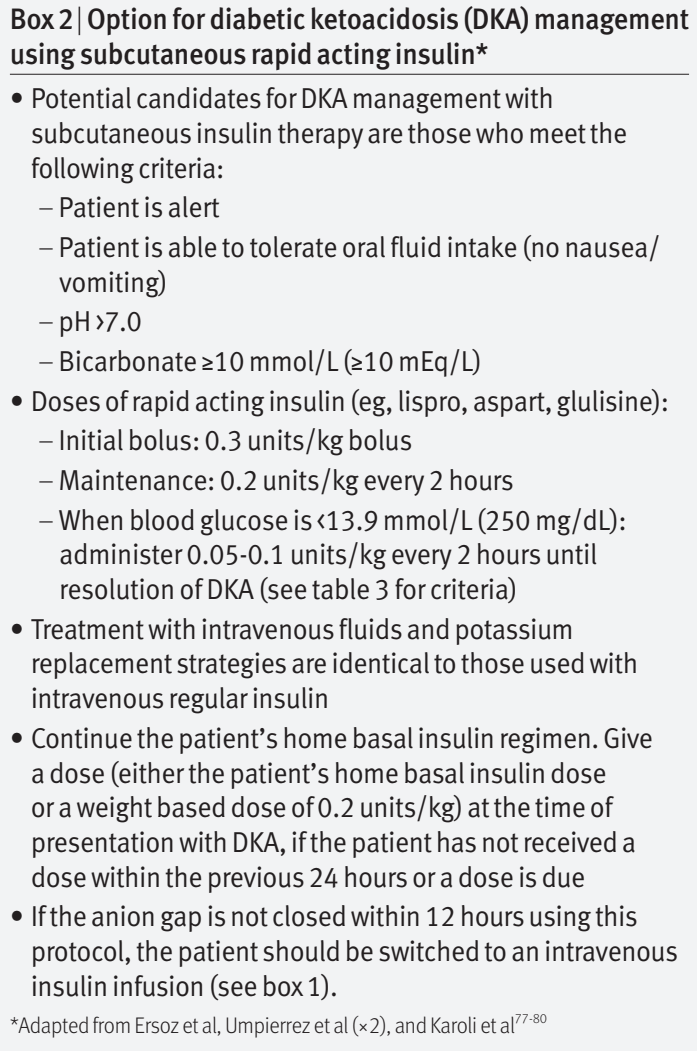

have them they are often not available 24 hours a day. ${ }^{41}$ In addition, blood ketones above $3 \mathrm{mmol} / \mathrm{L}$ ( $>31 \mathrm{mg} / \mathrm{dL}$ ) cannot be reliably measured using currently available devices, and measurements of decrements of $0.5 \mathrm{mmol} /$ $\mathrm{L} / \mathrm{h}(5.2 \mathrm{mg} / \mathrm{dL} / \mathrm{h})$ may be beyond the coefficient of variation of existing meters. ${ }^{6273}$ Given this, the UK guideline provides alternatives for situations in which bedside measurement of ketones is not possible. Specifically, the UK guideline recommends increasing insulin infusion rates by 1 unit/hour to achieve increases of bicarbonate concentrations at a rate of at least $3 \mathrm{mmol} / \mathrm{L} / \mathrm{h}(>3 \mathrm{mEq} /$ $\mathrm{L} / \mathrm{h}$ ) or decreases in blood glucose by at least $3 \mathrm{mmol} / \mathrm{L} / \mathrm{h}$ $(>50 \mathrm{mg} / \mathrm{dL} / \mathrm{h}){ }^{2}$ Of note, bicarbonate concentrations may not be reliable after the first six hours owing to the hyperchloremia resulting from continued use of normal saline.

\section{Concurrent basal insulin}

The UK guideline recommends continuing a patient's usual basal insulin dose or starting weight based basal insulin during acute diabetic ketoacidosis management. ${ }^{2}$ In patients not previously treated with insulin, the UK guideline recommends starting basal insulin at a dose of 0.25 units $/ \mathrm{kg}^{2}$ This approach is supported by a small RCT that compared 31 patients treated with intravenous insulin and 30 patients treated with intravenous insulin plus 0.25 units/ $\mathrm{kg}$ of insulin glargine given within 12 hours of starting the intravenous insulin. ${ }^{74}$ Patients receiving basal insulin had less rebound hyperglycemia without increased hypoglycemia in the 12 hours after discontinuation of intravenous insulin. Another randomized controlled pilot study found trends toward a reduction in time to anion gap closure and length of stay in hospital with concurrent insulin glargine. ${ }^{75}$
Use of subcutaneous rapid acting insulin

An alternative to intravenous insulin therapy for acute management of mild-moderate diabetic ketoacidosis is the use of subcutaneous rapid acting insulin (box 2).$^{76}$ Potential candidate patients include those who are alert and do not otherwise need admission to a critical care area, have a $\mathrm{pH}$ above 7.0, and bicarbonate of at least $10 \mathrm{mmol} / \mathrm{L}(\geq 10$ $\mathrm{mEq} / \mathrm{L}) .^{77}$ Four prospective randomized studies in adult patients with diabetic ketoacidosis compared subcutaneous rapid acting insulin (initial bolus of 0.3 units/ $\mathrm{kg}$ followed by 0.1-0.2 units/kg every 1-2 hours) with conventional diabetic ketoacidosis treatment and found no difference in patient outcomes. ${ }^{77-80}$ With subcutaneous insulin regimens, administration of intravenous fluids and electrolytes, as well as monitoring, is identical to what is recommended with intravenous insulin therapy. Factors that may affect the efficacy of subcutaneous insulin regimens include potential absorption problems in patients who are severely dehydrated, possibility of missed insulin doses, and altered pharmacokinetics of subcutaneous compared with intravenous insulin.

\section{Phosphate replacement}

Patients with diabetic ketoacidosis also have total body phosphate deficits, but no prospective studies have shown that phosphate replacement improves outcome. Insulin therapy will decrease phosphate, but overcorrection with phosphate can cause hypocalcemia. The UK guideline recommends against routine phosphate replacement; checking and replacing phosphate should occur only if the patient has symptoms of respiratory and skeletal muscle weakness. ${ }^{2}$ The ADA recommends that 20-30 mmol of phosphate may be indicated in patients with cardiac dysfunction, anemia, respiratory depression, or a phosphate concentration below $0.32 \mathrm{mmol} / \mathrm{L}$ $(<1 \mathrm{mg} / \mathrm{dL}){ }^{1}$

\section{Use of sodium bicarbonate}

A systematic review found that the available evidence, which includes three RCTs, does not support replacing bicarbonate in adult diabetic ketoacidosis patients with a $\mathrm{pH}$ of 6.9 or higher. ${ }^{81}$ The review also found retrospective evidence of transient paradoxical worsening of ketosis and an increased need for potassium supplementation in patients who received bicarbonate. ${ }^{81}$ Owing to possible harm and lack of sustained benefits, the UK guideline does not recommend the use of bicarbonate in any patients with diabetic ketoacidosis. ${ }^{2}$ On the other hand, as the use of bicarbonate has not been prospectively studied in patients with a $\mathrm{pH}$ below 6.9, and these patients are at very high risk for poor outcome, the ADA guideline recommends that slow administration of $100 \mathrm{mmol}(100$ $\mathrm{mEq}) \mathrm{NaHCO}_{3}$ over two hours be considered when the $\mathrm{pH}$ is below 6.9. ${ }^{1}$

\section{Management of HHS}

The goals of HHS treatment include correction of volume deficits while reducing and normalizing plasma hyperosmolality, which will correct hyperglycemia (box 3). Patients with HHS always need to be admitted to hospital, not only to achieve metabolic stability, which often requires more than 24 hours of treatment, but also to identify and treat the precipitating 
Box 3 Principles of hyperosmolar hyperglycemic syndrome (HHS) management in adult patients

\section{Intravenous fluids}

1 Use $1000 \mathrm{~mL}$ of $0.9 \%$ sodium chloride solution (normal saline) over 1 hour for initial fluid replacement

2 After the first hour, the rate of intravenous fluids should be adjusted on the basis of the patient's hemodynamic and electrolyte status and generally maintained between 250 and $500 \mathrm{~mL} / \mathrm{h}$

- ADA: Patients with a normal or high corrected sodium can be switched to $0.45 \%$ sodium chloride after the first hour of fluid replacement

- UK: Adjust hourly rate of normal saline to achieve a decline in blood glucose of 4-6 $\mathrm{mmol} / \mathrm{L} / \mathrm{h}(70-100 \mathrm{mg} / \mathrm{dL} / \mathrm{h})$ and plasma osmolality of 3-8 $\mathrm{mOsmol} / \mathrm{kg} / \mathrm{h}$. Switch from normal saline to $0.45 \%$ sodium chloride solution only when the fall in plasma osmolality and plasma glucose plateaus in the setting of adequate positive fluid balance

3 Add dextrose to the intravenous fluid if/when blood glucose approaches normal to allow continued insulin infusion at a rate sufficient to resolve diabetic ketoacidosis while avoiding hypoglycemia

- ADA: Add dextrose $5 \%$ when the blood glucose falls below $16.7 \mathrm{mmol} / \mathrm{L}(300 \mathrm{mg} / \mathrm{dL})$

- UK: Add dextrose $5 \%$ or dextrose $10 \%$ when the blood glucose falls below $14 \mathrm{mmol} / \mathrm{L}$ $(250 \mathrm{mg} / \mathrm{dL})$

\section{Electrolytes}

1 Patients with HHS have total body potassium deficits that must be replaced after adequate renal function (urine output) is assessed

- ADA: 20-30 mmol (20-30 mEq) potassium in each liter of infusion fluid when serum potassium is $<5.2 \mathrm{mmol} / \mathrm{L}(<5.2 \mathrm{mEq} / \mathrm{L})$

- UK: $40 \mathrm{mmol} / \mathrm{L}(40 \mathrm{mEq})$ in each liter of normal saline when serum potassium is $<5.5$ $\mathrm{mmol} / \mathrm{L}(\ll 5.5 \mathrm{mEq} / \mathrm{L})$ and the patient is passing urine

- Evidence is lacking to support either recommendation over the other

- Note: Because insulin therapy promotes an intracellular shift of potassium, it is recommended that insulin (if used) should not be started if the serum potassium is $<3$ $\mathrm{mmol} / \mathrm{L}(\ll 3 \mathrm{mEq} / \mathrm{L})$ to avoid worsening of hypokalemia

2 Routine replacement of phosphate is not recommended

Insulin

1 The optimal time to start intravenous insulin in the management of HHS has not been determined

- ADA: Start intravenous insulin after initiation of fluid resuscitation and correction of any hypokalemia

- UK: Start intravenous insulin if patient has $3-\beta$-hydroxybutyrate concentrations $>1.0$ $\mathrm{mmol} / \mathrm{L}(>10.4 \mathrm{mg} / \mathrm{dL}$ ) (or $>1.5 \mathrm{mmol} / \mathrm{L}(>15.6 \mathrm{mg} / \mathrm{dL}) 82)$. Otherwise, intravenous insulin can be started once blood glucose is falling below $5 \mathrm{mmol} / \mathrm{L} / \mathrm{h}(190 \mathrm{mg} / \mathrm{dL} / \mathrm{h})$ in the setting of adequate positive fluid balance.

2 The optimal starting dose of intravenous insulin in the management of HHS has not been determined

- ADA: Start intravenous insulin at either a fixed weight based dose of $0.14 \mathrm{units} / \mathrm{kg} / \mathrm{h}$ or at a fixed weight based dose of 0.1 units $/ \mathrm{kg} / \mathrm{h}$ after a 0.1 units $/ \mathrm{kg}$ bolus

- UK: Start low dose intravenous insulin (0.05 units $/ \mathrm{kg} / \mathrm{h}$ )

3 The rate of intravenous insulin should be adjusted to achieve an adequate drop in blood glucose

- ADA: If plasma glucose does not decrease by 3-4 mmol/L/h $(50-75 \mathrm{mg} / \mathrm{dL} / \mathrm{h})$ from the initial value in the first hour, the insulin infusion should be increased every hour until a steady glucose decline is achieved. When blood glucose is below $16 \mathrm{mmol} / \mathrm{L}(1300 \mathrm{mg} /$ $\mathrm{dL}$ ), adjust dextrose or intravenous insulin rate to maintain concentrations in the 14-16 $\mathrm{mmol} / \mathrm{L}(250-300 \mathrm{mg} / \mathrm{dL}$ ) range until HHS has resolved

- UK: Adjust insulin infusion rate hourly by 1 unit/h to maintain blood glucose decline of $<5 \mathrm{mmol} / \mathrm{L} / \mathrm{h}(<90 \mathrm{mg} / \mathrm{dL} / \mathrm{h})$, noting that blood glucose should not fall below $10-15$ $\mathrm{mmol} / \mathrm{L}(180-270 \mathrm{mg} / \mathrm{dL})$ during the first 24 hours of management

Identify/treat reason for HHS and prevent recurrence

See box 4

$A D A=$ recommendations in the American Diabetes Association guideline ${ }^{1}$.

UK=recommendation in the Joint British Diabetes Societies for Inpatient Care guideline ${ }^{4}$ cause. The ADA has not published a separate guideline for the management of HHS and includes recommendations for both diabetic ketoacidosis and HHS in the same document. ${ }^{1}$ The Joint British Diabetes Societies for Inpatient Care have a dedicated document for the management of HHS. ${ }^{4}$ Of note, virtually no published studies exist to guide the optimal treatment of HHS, which has resulted in differences in the ADA and UK guidelines. ${ }^{63}$

Intravenous fluids

Intravenous fluids are the first line of treatment in HHS to normalize osmolality, restore intravascular volume, and lower blood glucose. ADA and UK guidelines for HHS management both recommend at least $1 \mathrm{~L}$ of normal saline during the first hour, followed by adjustment of the infusion rate on the basis of the patient's hemodynamic and electrolyte status and the achievement of a positive fluid balance.

The ADA generally recommends $250-500 \mathrm{~mL} / \mathrm{h}$ (after the first hour) in adult patients without cardiac or renal compromise, advanced liver disease, and other states of fluid overload. The ADA recommends a switch to $0.45 \%$ sodium chloride if sodium concentrations are high and the addition of dextrose $5 \%$ when blood glucose reaches $16.7 \mathrm{mmol} / \mathrm{L}(300 \mathrm{mg} / \mathrm{dL}){ }^{1}$ The UK guideline recommends adjusting the hourly rate of normal saline to achieve a decline in blood glucose of 4-6 mmol/L/h (70$100 \mathrm{mg} / \mathrm{dL} / \mathrm{h}$ ) and plasma osmolality of $3-8 \mathrm{mOsmol} /$ $\mathrm{kg} / \mathrm{h} .{ }^{4}$ The UK guideline recommends switching from normal saline to $0.45 \%$ sodium chloride solution only when the fall in plasma osmolality and plasma glucose plateaus in the setting of adequate positive fluid balance. The UK guideline recommends adding dextrose 5-10\% to intravenous fluids when the blood glucose falls below $13.9 \mathrm{mmol} / \mathrm{L}$ (250 mg/dL).

\section{Potassium and phosphate replacement}

No studies have investigated the optimal approach to treatment of hypokalemia or hypophosphatemia in HHS patients; however, patients with HHS typically have more severe total body depletion than diabetic ketoacidosis patients, and close monitoring is advised. The ADA recommends adding 20-30 mmol (20-30 $\mathrm{mEq})$ potassium in each liter of infusion fluid when serum potassium is below $5.2 \mathrm{mmol} / \mathrm{L}(5.2 \mathrm{mEq} / \mathrm{L}){ }^{1}$ By contrast, the UK guideline recommends $40 \mathrm{mmol} / \mathrm{L}(40 \mathrm{mEq} / \mathrm{L})$ in each liter of normal saline when serum potassium is below 5.5 $\mathrm{mmol} / \mathrm{L}(5.5 \mathrm{mEq} / \mathrm{L})$ and the patient is passing urine. Because insulin therapy promotes an intracellular shift in potassium, it is recommended that insulin should not be started if the serum potassium is below $3 \mathrm{mmol} / \mathrm{L}$ ( $3 \mathrm{mEq} / \mathrm{L}$ ) to avoid worsening of hypokalemia. The UK guideline recommends replacing phosphate if hypophosphatemia persists beyond the acute phase of treatment of HHS. ${ }^{4}$

Insulin

The question of when to start insulin administration in the management of HHS has not been formally studied. The ADA guideline recommends starting intravenous regular insulin in the same way as during diabetic ketoaci- 


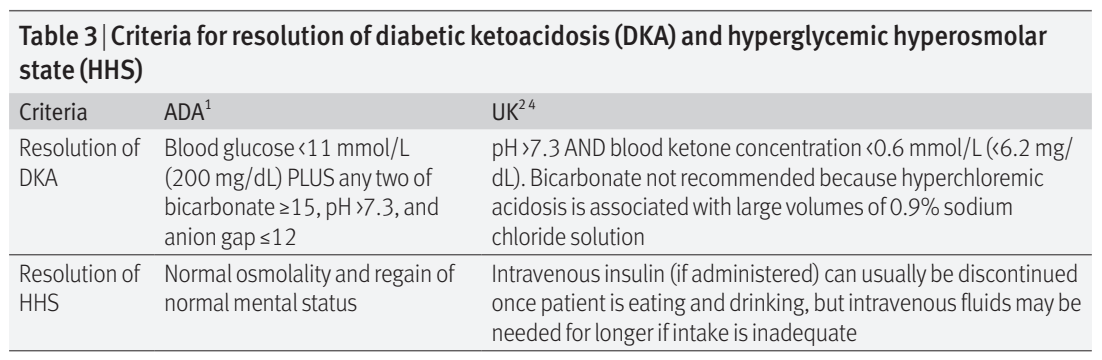

dosis management. That is, starting intravenous regular insulin at either a fixed weight based dose of 0.14 units/ $\mathrm{kg} / \mathrm{h}$ or at a fixed weight based dose of 0.1 units $/ \mathrm{kg} / \mathrm{h}$ followed by a 0.1 units $/ \mathrm{kg}$ bolus of intravenous insulin after initiation of fluid resuscitation and correction of any hypokalemia. ${ }^{1}$ The ADA recommends reducing insulin infusion rates to $0.02-0.05 \mathrm{units} / \mathrm{kg} / \mathrm{h}$ at the same time that dextrose $5 \%$ is added to the intravenous fluids when blood glucose declines to below $16.7 \mathrm{mmol} / \mathrm{L}$ (300 mg/ dL) in patients with HHS. ${ }^{1}$

On the other hand, the UK guideline recommends delaying intravenous insulin therapy unless the patient has 3- $\beta$-hydroxybutyrate concentrations above 1.0 $\mathrm{mmol} / \mathrm{L}(10.4 \mathrm{mg} / \mathrm{dL})$ or above $1.5 \mathrm{mmol} / \mathrm{L}(15.6 \mathrm{mg} /$ dL). ${ }^{482}$ The rationale for this recommendation is that early initiation of insulin in the setting of inadequate fluid replacement can aggravate hypoperfusion with an increase in risk for circulatory compromise and thrombosis, and intravenous fluid alone will reduce hyperglycemia. ${ }^{4}$ The ideal decline in blood glucose is less than $5 \mathrm{mmol} / \mathrm{L} / \mathrm{h}(<90 \mathrm{mg} / \mathrm{dL} / \mathrm{h})$, and concentrations should not fall below $10-15 \mathrm{mmol} / \mathrm{L}$ (180-270 mg/dL) during the first 24 hours of management. If the blood glucose fails to decline despite adequate fluids, then the recommended fixed rate of intravenous insulin is lower ( 0.05 units $/ \mathrm{kg}$ ) than is recommended for diabetic ketoacidosis. ${ }^{4}$

\section{Transition from acute management}

After resolution of diabetic ketoacidosis and HHS (table 3), all patients need to be transitioned from intravenous to subcutaneous insulin. ${ }^{167}$ This includes patients with euglycemic diabetic ketoacidosis secondary to treatment with an SGLT2 inhibitor and those with ketosis prone diabetes who present with diabetic ketoacidosis. To prevent rebound ketoacidosis or hyperglycemia, administration of a long acting basal insulin (if this has not already been given in the previous 24 hours) with or without a short or rapid acting insulin is needed at least two hours before the intravenous insulin infusion is stopped. ${ }^{67745}$ This overlap is needed mainly because of the short half life of intravenous insulin (approximately 10 minutes). ${ }^{7}$ When this transition is timed before a meal, administration of a prandial dose of short or rapid acting insulin together with the basal insulin may allow discontinuation of intravenous insulin in an hour. ${ }^{82}$ The ADA and UK guidelines recommend that patients previously treated with subcutaneous insulin can be restarted on their pre-admission insulin doses if these are determined to be appropriate. ${ }^{12}$ Otherwise, a weight based subcutaneous insulin regimen can be started by calculating a total dose of 0.5-0.7 units $/ \mathrm{kg} / \mathrm{d}$ and giving $50 \%$ of the total dose as once daily basal insulin and dividing the other $50 \%$ equally
Box 4 | Identification and treatment of underlying causes and prevention of recurrence of diabetic ketoacidosis or hyperosmolar hyperglycemic syndrome

1 Assess and manage reasons for insulin non-adherence (if applicable)

- Consider alternative (human) insulin regimens and/ or social work consultation if the patient is unable to afford the prescribed insulin

- Facilitate treatment or social services for patients with untreated or poorly controlled psychiatric disorders

- Refer patients with substance or alcohol use disorders to treatment programs

2 Provide education on appropriate sick day management (if applicable) and refer the patient to a formal diabetes education program after hospital discharge

3 Treat treatable precipitating causes such as infection, cerebrovascular accident, myocardial infarction, or trauma

between pre-breakfast, pre-lunch, and pre-supper doses of rapid acting insulin. ${ }^{127}$

Patients who meet metabolic criteria for resolution of the diabetic crisis (table 3), but who remain critically ill and unable to tolerate oral intake, will need ongoing management with intravenous fluids and insulin, the latter titrated using an insulin infusion protocol with prespecified glycemic targets for critical illness. ${ }^{83} 84$

\section{Prevention of diabetic ketoacidosis and HHS}

Recurrent episodes of diabetic ketoacidosis and HHS can be prevented in many cases. ${ }^{85}$ The cause of every episode of diabetic ketoacidosis and HHS should be determined so that tailored education and intervention can be provided to the patient to prevent recurrence (box 4). ${ }^{85} 86$ To prevent readmission to hospital and visits to the emergency department, education based on the needs of the individual patient should be provided before discharge. ${ }^{86}$ This education, sometimes called "survival skills" education, can include a review of the causes, signs, and symptoms of impending diabetic ketoacidosis, as well as what to do and who and when to call when symptoms develop. Providing patients and their family members with information on how to manage their diabetes drugs during periods of acute illness can reduce the metabolic decompensation that can occur with inappropriate reductions or omissions of insulin doses.$^{87}$ In addition, ensuring that patients have access to their diabetes drugs, including the ability to pay for these drugs, is important in preventing readmissions. ${ }^{88} 89$

All patients with diabetes should receive initial and ongoing education about diabetes in the community setting, as this can help to prevent diabetes related hospital admissions (and readmissions). This education is best provided or directed by a trained diabetes educator. ${ }^{86} 89$ ${ }^{90}$ Patient specific instructions for sick day management, including reminders that intermediate or long acting insulin should never be stopped and when to call for assistance, can help to prevent future episodes of diabetic ketoacidosis. ${ }^{9192}$ All patients with type 1 diabetes and many insulin treated patients with type 2 diabetes should have a means to measure ketones at home and be instructed to monitor both glucose and ketones at least every four hours during illness. 
To minimize the risk of euglycemic diabetic ketoacidosis with SGLT2 inhibitors, patients can be educated to stop these drugs for at least 24 hours and up to five days before planned surgical procedures or during periods of acute illness. ${ }^{5750}$ The half life of this class of agents is approximately 13 hours, but the clinical effect can last for several days after discontinuation. ${ }^{47-50} 93$ Patients need to be educated about situations that predispose them to developing euglycemic diabetic ketoacidosis, including dehydration or ingestion of excessive amounts of alcohol. ${ }^{54750}$ Patients taking SGLT2 inhibitors should be instructed to check for ketones during times of illness and metabolic stress. When high ketone concentrations are detected, a proposed strategy to prevent progression to diabetic ketoacidosis is to stop the SGLT2 inhibitor, inject bolus insulin, consume $30 \mathrm{~g}$ of carbohydrate, hydrate with water, and continue to monitor ketones every three to four hours. ${ }^{50}$ Patients should be educated on potential symptoms heralding the onset of diabetic ketoacidosis and be instructed to seek care if they develop these symptoms, regardless of their blood glucose concentration.

\section{Complications related to management of diabetic ketoacidosis and HHS}

Several complications can be associated with diabetic crises and their management.

\section{Cerebral edema}

Of these complication, the development of cerebral edema is the most serious. ${ }^{1194}$ This has been described most commonly in young children and adolescents presenting with diabetic ketoacidosis as the initial manifestation of new onset type 1 diabetes, but it has also been described in young adults up to age $28 .^{9495}$

Rare cases of cerebral edema occur in adults over age 28 , but current recommendations suggest maintaining blood glucose concentration no lower than 13.9-16.6 $\mathrm{mmol} / \mathrm{L}$ (250-300 $\mathrm{mg} / \mathrm{dL}$ ) for several hours during the course of treatment for patients with either diabetic ketoacidosis or HHS as a potential method for avoiding this devastating complication. ${ }^{127}$

Early recognition of potential neurologic deterioration such as new onset or intensifying headache, a decline in level of consciousness, recurrent vomiting, incontinence, irritability, abnormal respirations, a delayed rise in serum sodium with treatment, or evidence of cranial nerve dysfunction provide suggestive evidence of onset of cerebral edema. Prompt administration of mannitol therapy administered at a dose of $0.5-1 \mathrm{~g} / \mathrm{kg}$ over 20 minutes can help to abort further neurologic deterioration. ${ }^{96}$ Delaying potential lifesaving treatment while awaiting results of computed tomography imaging is not recommended.

Modification of the rate of intravenous fluid administration has previously been suggested as a potential method for ameliorating this risk. A recent multicenter trial that included 1255 children with diabetic ketoacidosis randomly assigned to one of four treatment groups receiving $0.45 \%$ or $0.9 \%$ sodium chloride containing intravenous fluids administered at a slow or rapid rate showed no difference in neurologic outcomes. ${ }^{95}$

\section{Electrolyte abnormalities}

More commonly observed complications in adults include hypokalemia and hyperkalemia, hypoglycemia, and non-anion gap hyperchloremic metabolic acidosis. ${ }^{415667}$ Hypokalemia is reported more frequently than hyperkalemia and usually results from delays in administration of or insufficient potassium containing supplementation. Hyperkalemia can result from overly aggressive potassium replacement, particularly in patients with underlying renal dysfunction. ${ }^{5567}$ Hypoglycemia can result from overly aggressive insulin infusions, insufficient frequency of blood glucose monitoring, or failure to add dextrose to intravenous fluids when blood glucose concentrations approach $13.9 \mathrm{mmol} / \mathrm{L}(250 \mathrm{mg} / \mathrm{dL}){ }^{5556}$

In a retrospective review comparing 8550 adult patients with diabetic ketoacidosis admitted to an intensive care unit where blood glucose was corrected to 10 $\mathrm{mmol} / \mathrm{L}$ (180 mg/dL) or lower or to above $10 \mathrm{mmol} / \mathrm{L}$ (180 $\mathrm{mg} / \mathrm{dL}$ ) within 24 hours, those in the latter group had less hypoglycemia, hypokalemia, and hypo-osmolality, with lower mortality. ${ }^{97}$ However, the fact that these complications still occurred in approximately $25 \%$ of patients treated with standardized protocols suggests the need to monitor electrolytes and blood glucose concentrations no less often than every two hours while intravenous insulin infusions are continued. In a national survey from the UK, suboptimal adherence to hospital based protocols and national guidelines was identified as a major contributor to these frequently observed complications. ${ }^{4142}$ Although efforts are under way in some institutions to mitigate these complications through modification of existing protocols, attention to staff education and ongoing monitoring or protocol adherence may also help to reduce these risks. ${ }^{67}$

The development of non-anion gap hyperchloremic metabolic acidosis often follows the acute phase of diabetic ketoacidosis management. ${ }^{13498}$ This has been attributed to administration of large volumes of intravenous fluids containing normal saline during acute diabetic ketoacidosis management, as well as to urinary losses of keto-anions that are needed for regeneration of bicarbonate. This is not considered to be a serious complication and usually resolves spontaneously in a few days. ${ }^{198}$ No evidence supports the use of colloid solutions, balanced electrolyte solutions (such as Plasma-Lyte), or Ringer's lactate in place of $0.9 \%$ sodium chloride solution in the management of diabetic ketoacidosis. ${ }^{60}$ In one RCT conducted at two institutions, 57 patients admitted with diabetic ketoacidosis were randomly assigned to receive fluid resuscitation with $0.9 \%$ sodium chloride or Ringer's lactate. It found no difference in time to resolution of diabetic ketoacidosis, with a delay in achieving blood glucose concentrations below $14 \mathrm{mmol} / \mathrm{L}$ $(252 \mathrm{mg} / \mathrm{dL})$ in the group receiving Ringer's lactate. ${ }^{99}$ In another RCT, 45 adult patients with type 1 diabetes admitted with diabetic ketoacidosis were randomly assigned to standardized resuscitation with normal saline or a balanced electrolyte solution with measurement of serum chloride and bicarbonate every two hours for 12 hours. Those receiving the balanced electrolyte solution had lower chloride and higher bicarbonate concentrations, although the clinical significance remains to be determined. ${ }^{100}$ 
Cardiac, respiratory, and muscle complications

Other complications that occur less frequently but for which monitoring is important include myocardial infarction, potential for pulmonary edema in patients with underlying congestive heart failure, and rhabdomyolysis in patients who present with more severe degrees of dehydration. ${ }^{101}$ Awareness is also increasing that complications of diabetic ketoacidosis may continue beyond the acute hospital admission. In one recent study of 3572 patients with type 2 diabetes and 7144 controls matched for age, sex, and baseline diabetes complications and comorbidities, patients with diabetic ketoacidosis were 1.55 times more like to experience a stroke within six months than were those without diabetic ketoacidosis. ${ }^{102}$ In another study examining long term outcomes in patients admitted to an intensive care unit with diabetic ketoacidosis, one in 10 patients died within one year of hospital discharge. ${ }^{103}$ The average age of patients in this report was 38 years, suggesting that these patients represent a group for whom a heightened degree of surveillance with associated interventions is needed to offset the mortality risk.

\section{Recurrent diabetic ketoacidosis}

Patients admitted to hospital with diabetic ketoacidosis represent a group at high risk for all cause mortality and hospital readmissions including recurrent diabetic ketoacidosis. ${ }^{13103-105}$ Those with a history of psychiatric illness or alcohol or substance misuse are at even higher risk for readmission, which contributes to long term risk for death. ${ }^{106} 107$ Interventions designed to improve a patient's compliance with diabetes treatment and help to reduce the risk for recurrent diabetic ketoacidosis, such as those described in the prevention section of this paper, are essential.

\section{Summary of guidelines}

The ADA published a consensus statement guiding treatment for adult patients with hyperglycemic crises in 2009. ${ }^{1}$ Although not specifically designated as a guideline, it has been used for this purpose with several more recent reviews and commentaries that outline both the strengths and weaknesses of this widely followed report, as well as areas that are in need of revision or future study..$^{7106063}$ The most recent 2019 ADA clinical practice recommendations for management of diabetic ketoacidosis include several updates to the 2009 consensus statement, as pointed out in table 1 and table $2 .{ }^{6}$ The UK guidelines for management of diabetic ketoacidosis and HHS, written by the Joint British Diabetes Societies Inpatient Care Group and most recently updated in 2013 and 2016, respectively, have also been subject to similar scrutiny to those of the ADA. ${ }^{24}$

The sections on treatment of diabetic ketoacidosis and HHS included in this manuscript provide direct comparisons of recommendations of the ADA and UK guidelines for fluid resuscitation, electrolyte repletion, insulin therapy, and transitioning patients back to a home regimen of glycemic management where this is appropriate. No studies have compared strategies recommended by the ADA and UK, and we believe that this type of study is not necessary as the subtle differences in rate of intravenous fluid administration, electrolyte replacement, or dosing of intravenous insulin are not likely to result in major differences in outcomes. One difference in the guidelines for diabetic ketoacidosis that may need additional investigation is continuation of the home insulin regimen during the acute management of diabetic ketoacidosis. The UK guideline recommends continuation of basal insulin; however, administration of basal insulin remains an area of investigation in the US, as it may be associated with increased rates of hypoglycemia when used concurrently with the recommended rates of intravenous insulin. An additional area that needs further investigation is the use of blood ketones to adjust the rate of intravenous insulin in the management of diabetic ketoacidosis, as is recommended in the UK guidelines but not widely adopted in the US owing to limitations and availability of point of care blood ketone meters. A third area needing further investigation is when intravenous insulin is indicated in the acute management of pure HHS without any evidence of elevated ketones. The UK guidelines do not recommend routine use of intravenous insulin in the initial management of HHS.

The weakness of any guideline is in the implementation. Evidence suggests that guidelines for diabetic ketoacidosis and HHS are not consistently followed by many institutions in the US as well as the UK. Efforts to improve adherence to these guidelines may result in improved outcomes for patients and reductions in costs of care. ${ }^{4167}$

Also included in this manuscript is a discussion of euglycemic diabetic ketoacidosis with use of the SGLT2 inhibitor class of drugs. The only published statement for euglycemic diabetic ketoacidosis with this class of drugs was published as a position statement in 2016 by the American Association of Clinical Endocrinologists. ${ }^{5}$ For this disorder, education of patients and healthcare providers about recognition that this may be occurring is important to avoid significant patient morbidity.

\section{Emerging treatments}

Several studies are investigating optimal treatment strategies with insulin and intravenous fluids, as well as alternative treatment strategies and previously unexplored adverse outcomes in adult patients presenting with decompensated diabetes. Some of these trials are listed below.

Clinicaltrials.gov NCT02930044-The purpose of this study is to determine whether adult patients with diabetic ketoacidosis who present to the emergency department and are treated with glargine insulin ( 0.3 units/ $\mathrm{kg}$ with a maximum dose of 30 units) within two hours after starting the intravenous insulin infusion need a shorter duration of intravenous insulin administration to resolve diabetic ketoacidosis compared with standard care (insulin glargine administered two to three hours before stopping intravenous insulin). This study will provide additional information on use of long acting insulin preparations early in the course of treatment for diabetic ketoacidosis. ${ }^{745}$ It is scheduled to be completed in November 2019. 


\section{QUESTIONS FOR FUTURE RESEARCH}

1 Can patients at risk for euglycemic diabetic ketoacidosis with use of sodium glucose cotransporter 2 inhibitors be identified before these drugs are administered?

2 What are the optimal methods for continuation of intravenous insulin therapy that minimize risk for hypoglycemia?

3 What type of fluid replacement strategies would allow for correction of dehydration without resultant hyperchloremic metabolic acidosis?

4 Does a therapeutic approach exist that would further minimize the risk of cerebral edema as a complication of diabetic ketoacidosis in young adults?

5 What interventions effectively reduce the risk of recurrent diabetic ketoacidosis in patients who have frequent hospital readmissions?

\section{HOW PATIENTS WERE INVOLVED IN THE CREATION OF THIS MANUSCRIPT}

We invited three people with type 1 diabetes and a personal previous history of diabetic ketoacidosis who were known to us to review advanced drafts of this manuscript. We asked them for their comments about what they felt were areas that were missed and what may have been correctly or incorrectly emphasized. The consensus among these reviewers was that an emphasis was needed on the patient and family experience of decompensated diabetes as well as on prevention of recurrent episodes. All reported either personal or observed episodes of insulin doses being erroneously withheld during a hospital admission. Another suggestion was to emphasize the need to recognize diabetic ketoacidosis in both older and younger adult patients. The patient reviewers suggested emphasizing education on sick day management strategies such as checking for urine or blood ketones and preventing deterioration from ketosis or severe hyperglycemia due to diabetic ketoacidosis or hyperosmolar hyperglycemic syndrome (HHS), respectively. In addition, they recommended an emphasis on providing information on what to expect over the course of treatment for diabetic ketoacidosis and HHS as a way of decreasing fear and anxiety in patients and their families. As a result of their input, we clarified several areas of the manuscript and gave additional emphasis to other areas.

Clinicaltrials.gov NCT03717896-The purposes of this randomized, double blind, placebo controlled trial are to determine whether administration of intravenous thiamine (200 mg in normal saline twice daily for two days) will lead to quicker resolution of acidosis in patients with diabetic ketoacidosis and to investigate whether thiamine improves cellular oxygen consumption, shortens length of stay in hospital, or decreases resource use. This novel study is based on preliminary studies from the investigators showing that thiamine concentrations, which are deficient in up to $37 \%$ of patients with diabetic ketoacidosis, are inversely associated with the severity of acidosis. It is scheduled to be completed in 2023.

Clinicaltrials.gov NCT02172092-The purpose of this study is to assess shifts in blood and urine concentrations of sodium, chloride, and hemoglobin during fluid resuscitation in patients with diabetic ketoacidosis. It is scheduled to be completed in December 2019.

Clinicaltrials.gov NCTO2864914-This study aims to assess the risk of acute liver or kidney injury, urinary tract or genital infections, and diabetic ketoacidosis in patients treated with empagliflozin (a SGLT2 inhibitor) or a dipeptidyl peptidase 4 inhibitor. It is scheduled to be completed in 2020.

Clinicaltrials.gov NCT02443415-The purpose of this study, which has been completed (2017) but not yet published, was to evaluate changes in brain and memory function using cognitive testing and magnetic resonance imaging in patients with diabetes who have no history of diabetic ketoacidosis or who have one episode or more than three episodes of diabetic ketoacidosis. Healthy patients without diabetes were also studied.

\section{Conclusions}

Diabetic ketoacidosis and HHS are serious metabolic emergencies that have potential for adverse outcomes when not promptly recognized and treated. A worrisome increase in the incidence of admissions for diabetic ketoacidosis has occurred over the past two decades, with insulin omission and infection remaining the two predominant precipitating causes. ${ }^{121315}$ The introduction of the SGLT2 inhibitors has increased awareness of euglycemic diabetic ketoacidosis, which can occur with use of these agents as well as in other clinical situations. ${ }^{24}$ Educating patients to check urine or blood ketones in the presence of symptoms such as nausea, vomiting, and/or fatigue, even if blood glucose concentrations are below $11.1 \mathrm{mmol} / \mathrm{L}$ ( $200 \mathrm{mg} / \mathrm{dL}$ ), is important as these symptoms may represent early warning signs for diabetic ketoacidosis. ${ }^{50}$

Despite well written published guidelines for management of diabetic ketoacidosis and HHS, these guidelines are not always followed..$^{414267}$ This can result in rebound hyperglycemia, hypoglycemia, electrolyte abnormalities, and recurrence of diabetic ketoacidosis.$^{67}$ Additionally, common pitfalls in management include premature termination of intravenous insulin therapy and insufficient timing or dosing of subcutaneous insulin before discontinuation of intravenous insulin.

There is increasing recognition that a large percentage of patients who are admitted with diabetic ketoacidosis are a group at high risk for other life threatening events, mortality, and hospital readmission. ${ }^{103107}$ Strategies to prevent hospital readmissions, as well as long term morbidity and mortality, include engagement with diabetes educators who can educate patients on strategies for managing blood glucose concentrations during periods of illness and instruct patients on when to call their physician or diabetes healthcare provider for additional information. ${ }^{86} \mathrm{~A}$ greater focus is needed to educate physicians and patients and their families about early recognition, causes, and strategies for prevention of acute decompensated diabetes.

We thank Paul Strumph, Linda F Fried, and Heba Ismail for reviewing an earlier version of this manuscript and for providing commentary that was helpful in making this a patient based review of an important topic. We also thank Kellie Antinori-Lent for her expert suggestions for the section on patient education.

Contributors: EKF did the primary literature review for this manuscript and wrote the sections on the epidemiology and pathophysiology of diabetic ketoacidosis and hyperosmolar hyperglycemic syndrome (HHS). ACD wrote the sections on acute management of diabetic ketoacidosis and $\mathrm{HHS}$ and carefully compared and contrasted published guidelines from the UK and US. MTK guided the writing of the full manuscript and assumed primary responsibility for the sections on clinical presentation, prevention, and complications. All authors reviewed all sections of the manuscript, providing suggestions for included content and references. 
Competing interests: We have read and understood the BMJ policy on declaration of interests and declare the following interests: none.

Provenance and peer review: Commissioned; externally peer reviewed.

1 Kitabchi AE, Umpierrez GE Miles IM. Fisher IN. Hyperglycemic crises in adult patients with diabetes. Diabetes Care 2009:32:1335-43. 10.2337/dc09-9032 pmid:19564476

2 Joint British Diabetes Societies Inpatient Care Group. The management of diabetic ketoacidosis in adults. 2nd edition. 2013. http://www. diabetologists-abcd.org.uk/JBDS/JBDS IP_DKA Adults Revised.pdf.

3 Pasquel Fl, Umpierrez GE. Hyperosmolar hyperglycemic state: a historic review of the clinical presentation, diagnosis, and treatment. Diabetes Care 2014;37:3124-31. 10.2337/dc14-0984 pmid:25342831.

4 Scott AR. Joint British Diabetes Societies (JBDS) for Inpatient Care JBDS hyperosmolar hyperglycaemic guidelines group. Management of hyperosmolar hyperglycaemic state in adults with diabetes. Diabet Med 2015·32.714-24.10.1111/dme.12757 pmid.25980647.

5 Handelsman Y, Henry RR, Bloomgarden ZT, et al. American Association of Clinical Endocrinologists and American College of Endocrinology Position Statement On The Association OfSGLT-2 Inhibitors and Diabetic Ketoacidosis. Endocr Pract 2016;22:753-62. 10.4158/EP161292. PS pmid:27082665

6 American Diabetes Association. 15. Diabetes Care in the Hospital: Standards of Medical Care in Diabetes-2019. Diabetes Care 2019;42(Suppl 1):S173-81. 10.2337/dc19-S015 pmid:30559241.

7 Umpierrez G, Korytkowski M. Diabetic emergencies ketoacidosis, hyperglycaemic hyperosmolar state and hypoglycaemia. Nat Rev Endocrinol 2016;12:222-32.10.1038 nrendo.2016.15 pmid:26893262

8 Wachtel TJ, Tetu-Mouradjian LM, Goldman DL, Ellis SE, O'Sullivan PS. Hyperosmolarity and acidosis in diabetes mellitus: a three-year experience in Rhode Island. J Gen Intern Med 1991;6:495-502. 10.1007/BF02598216 pmid:1765864

9 Munro JF, Campbell IW, McCuish AC, Duncan LJ. Euglycaemic diabetic ketoacidosis. BrMed J 1973;2:578-80. 10.1136/ bmj.2.5866.578 pmid:4197425.

10 Dhatariya KK, Umpierrez GE. Guidelines for management of diabetic ketoacidosis: time to revise? Lancet Diabetes Endocrinol 2017:5.321-3. 10.1016/S2213-8587(17)30093-1 pmid:28372975.

11 Troy PJ, Clark RP, Kakarala SG, Burns J, Silverman IE, Shore E. Cerebral edema during treatment of diabetic ketoacidosis in an adult with new onset diabetes. Neurocrit Care 2005;2:55-8. 10.1385 NCC:2:1:055 pmid:16174971

12 Benoit SR, Zhang Y, Geiss LS, Gregg EW, Albright A. Trends in Diabetic Ketoacidosis Hospitalizations and In-Hospital Mortality - United States, 2000-2014. MMWR Morb Mortal Wkly Rep 2018;67:362-5. 10.15585/ mmwr.mm6712a3 pmid:29596400.

13 Zhong VW, Juhaeri J, Mayer-Davis EJ. Trends in Hospital Admission for Diabetic Ketoacidosis in Adults With Type 1 and Type 2 Diabetes in England, 1998-2013: A Retrospective Cohort Study. Diabetes Care 2018;41:1870-7.10.2337/dc17-1583 pmid:29386248

14 Javor KA, Kotsanos JG, McDonald RC, Baron AD, Kesterson JG, Tierney WM Diabetic ketoacidosis charges relative to medical charges of adult patients with type I diabetes. Diabetes Care 1997:20:349-54. 10.2337 diacare.20.3.349 pmid:9051386.

15 Centers for Disease Control and Prevention. National Diabetes Statistics Report. 2017. https://www.cdc.gov/diabetes/data/statistics/statisticsreport.html.

16 Vellanki P. Umpierrez GE Diabetic ketoacidosis: A common debut of diabetes among african americans with type 2 diabetes. Endocr Pract 2017;23:971-8. 10.4158/EP161679.RA pmid:28534682.

17 Cefalu WT, Dawes DE, Gavlak G, et al. Insulin Access and Affordability Working Group: Conclusions and Recommendations. Diabetes Care 2018;41:1299-311.10.2337/dci18-0019 pmid:29739814.

18 Dhatariya KK, Skedgel C, Fordham R. The cost of treating diabetic ketoacidosis in the UK: a national survey of hospital resource use. Diabe Med 2017;34:1361-6. 10.1111/dme.13427 pmid:28727175

19 Delaney MF, Zisman A, Kettyle WM. Diabetic ketoacidosis and hyperglycemic hyperosmolar nonketotic syndrome. Endocrinol Metab Clin North Am 2000;29:683-705, V. 10.1016/S0889-8529(05)701596 pmid:11149157.

20 Fadini GP, de Kreutzenberg SV, Rigato M, et al. Characteristics and outcomes of the hyperglycemic hyperosmolar non-ketotic syndrome in cohort of 51 consecutive cases at a single center. Diabetes Res Clin Pract 2011:94:172-9. 10.1016/j.diabres.2011.06.018 pmid:21752485.

21 Peters AL, Buschur EO, Buse IB, Cohan P, Diner IC, Hirsch IB. Euglycemic Diabetic Ketoacidosis: A Potential Complication of Treatment With Sodium-Glucose Cotransporter 2 Inhibition. Diabetes Care 2015;38:1687-93.10.2337/dc15-0843 pmid:26078479.

22 Meyer EJ, Gabb G, Jesudason D. SGLT2 Inhibitor-Associated Euglycemic Diabetic Ketoacidosis: A South Australian Clinical Case Series and Australian Spontaneous Adverse Event Notifications. Diabetes Care 2018;41:e47-9. 10.2337/dc17-1721 pmid:29440112

23 Ueda P, Svanström H, Melbye M, et al. Sodium glucose cotransporter 2 inhibitors and risk of serious adverse events: nationwide register based cohor study. BM/ 2018;363:k4365. 10.1136/bmi.k4365 pmid:30429124.

24 Modi A, Agrawal A, Morgan F. Euglycemic Diabetic Ketoacidosis: A Review. Curr Diabetes Rev 2017;13:315-21. 10.2174/1573399812666160421121307 pmid:27097605
25 Nyenwe EA, Kitabchi AE. The evolution of diabetic ketoacidosis: An update of its etiology, pathogenesis and management. Metabolism 2016:65:50721.10.1016/j.metabol.2015.12.007 pmid:26975543.

26 Miles JM, Rizza RA, Haymond MW, Gerich JE. Effects of acute insulin deficiency on glucose and ketone body turnover in man: evidence for the primacy of overproduction of glucose and ketone bodies in the genesis of diabetic ketoacidosis. Diabetes 1980;29:926-30. 10.2337/ diab.29.11.926 pmid:6775996.

27 DeFronzo RA, Ferrannini E. Regulation of hepatic glucose metabolism in humans. Diabetes Metab Rev 1987;3:415-59.10.1002/ dmr.5610030204 pmid:3552529.

28 Exton JH. Mechanisms of hormonal regulation of hepatic glucose metabolism. Diabetes Metab Rev 1987;3:163-83.10.1002/ dmr.5610030108 pmid:3032541.

29 Meyer C, Stumvoll M, Dostou J, Welle S, Haymond M, Gerich J. Renal substrate exchange and gluconeogenesis in normal postabsorptive humans. Am J Physiol Endocrinol Metab 2002;282:E428-34. 10.1152/ ajpendo.00116.2001 pmid:11788376.

30 Meyer C, Stumvoll M Welle S, Woerle HI. Haymond M Gerich I. Relative importance of liver, kidney, and substrates in epinephrine-induced increased gluconeogenesis in humans. Am J Physiol Endocrinol Metab 2003;285:E819-26. 10.1152/ajpendo.00145.2003 pmid:12959936.

31 Foster DW, McGarry JD. The metabolic derangements and treatment of diabetic ketoacidosis. N Engl/ Med 1983;309:159-69.10.1056/ NEJM198307213090307 pmid:6408476

32 Miles JM, Haymond MW, Nissen SL, Gerich JE. Effects of free fatty acid availability, glucagon excess, and insulin deficiency on ketone body production in postabsorptive man. / Clin Invest 1983:71:1554-61. 10.1172/ICl110911 pmid:6134753.

33 Miles JM, Gerich JE. Glucose and ketone body kinetics in diabetic ketoacidosis. Clin Endocrinol Metab 1983;12:303-19. 10.1016/S0300595X(83)80043-7 pmid:6409465.

34 Palmer BF, Clegg DJ. Electrolyte and Acid-Base Disturbances in Patients with Diabetes Mellitus. NEngl/ Med 2015;373:548-59.10.1056/ NEJMra1503102 pmid:26244308.

35 McGarry JD, Foster DW. Regulation of hepatic fatty acid oxidation and ketone body production. Annu Rev Biochem 1980;49:395-420. 10.1146/ annurev.bi.49.070180.002143 pmid:6157353.

36 Dhatariya K. Blood Ketones: Measurement, Interpretation, Limitations, and Utility in the Management of Diabetic Ketoacidosis. Rev Diabet Stud 2016;13:217-25.10.1900/RDS.2016.13.217 pmid:28278308.

37 Umpierrez GE, KellyJP, Navarrete JE, Casals MM, Kitabchi AE. Hyperglycemic crises in urban blacks. Arch Intern Med 1997;157:669-75. 10.1001/ archinte.1997.00440270117011 pmid:9080921.

38 Randall L, Begovic J. Hudson M, et al. Recurrent diabetic ketoacidosis in innercity minority patients: behavioral, socioeconomic, and psychosocial factors. Diabetes Care 2011;34:1891-6. 10.2337/dc11-0701 pmid:21775761.

39 Lohiya S, Kreisberg R, Lohiya V. Recurrent diabetic ketoacidosis in two community teaching hospitals. Endocr Pract 2013;19:829-33. 10.4158/ EP13057.RA pmid:23757621.

40 Ananth J, Parameswaran S, Gunatilake S. Side effects of atypical antipsychotic drugs. Curr Pharm Des 2004;10:2219-29. 10.2174/1381612043384088 pmid.15281897.

41 Dhatariya K, Nunney I, Iceton G. Institutional factors in the management of adults with diabetic ketoacidosis in the UK: results of a national survey. DiabetMed 2016;33:269-70. 10.1111/dme.12877 pmid:26235104.

42 Dhatariya KK, Nunney I, Higgins K, Sampson MI, Iceton G. National survey of the management of Diabetic Ketoacidosis (DKA) in the UK in 2014. DiabetMed 2016;33:252-60.10.1111/dme.12875 pmid:26286235.

43 Polonsky WH, Anderson BJ, Lohrer PA, Aponte JE, Jacobson AM, Cole CF. Insulin omission in women with IDDM. Diabetes Care 1994:17:1178-85. 10.2337/diacare.17.10.1178 pmid:7821139.

44 Giessmann LC, Kann PH. Risk and Relevance of Insulin Pump Therapy in the Aetiology of Ketoacidosis in People with Type 1 Diabetes. Exp Clin Endocrinol Diabetes 2018.pmid:30049002.

45 Yogi-Morren D, Lansang MC. Management of patients with type 1 diabetes in the hospital. Curr Diab Rep 2014;14:458. 10.1007/s11892-0130458-9 pmid:24398661.

46 Blau JE, Tella SH, Taylor SI, Rother KI. Ketoacidosis associated with SGLT2 inhibitor treatment: Analysis of FAERS data. Diabetes Metab Res Rev 2017;33.10.1002/dmrr.2924 pmid:28736981.

47 Rosenstock J, Ferrannini E. Euglycemic Diabetic Ketoacidosis: A Predictable, Detectable, and Preventable Safety Concern With SGLT2 Inhibitors. Diabetes Care 2015:38:1638-42. 10.2337/dc151380 pmid:26294774

48 Taylor SI, Blau JE, Rother KI. SGLT2 Inhibitors May Predispose to Ketoacidosis. J Clin Endocrinol Metab 2015;100:2849-52. 10.1210/ jc. 2015-1884 pmid.26086329.

49 Hattersley AT, Thorens B. Type 2 Diabetes, SGLT2 Inhibitors, and Glucose Secretion. N Engl/ Med 2015;373:974-6.10.1056/ NEJMcibr1506573 pmid:26332554.

50 Garg SK, Peters AL, Buse JB, Danne T. Strategy for Mitigating DKA Risk in Patients with Type 1 Diabetes on Adjunctive Treatment with SGLT Inhibitors: A STICH Protocol. Diabetes Technol Ther 2018;20:571-5. 10.1089/dia.2018.0246 pmid:30129772. 
51 Wachtel TJ, Silliman RA, Lamberton P. Predisposing factors for the diabetic hyperosmolar state Arch Intern Med 1988:148.747.10.1001/ archinte.1988.00380030253040 pmid:3341875

52 Arieff Al, Carroll HJ. Cerebral edema and depression of sensorium in nonketotic hyperosmolar coma. Diabetes 1974;23:525-31. 10.2337/ diab.23.6.525 pmid:4834291.

53 Hara IS, Rahbar AJ, Jeffres MN, Izuora KE. Impact of a hyperglycemic crises protocol. Endocr Pract 2013;19:953-62. 10.4158/EP13077. OR pmid:23807521.

54 Bull SV, Douglas IS, Foster M, Albert RK. Mandatory protocol for treating adult patients with diabetic ketoacidosis decreases intensive care unit and hospital lengths of stay: results of a nonrandomized trial. Crit Care Med 2007:35:41-6. 10.1097/01.CCM.0000249825.18677. D2 pmid: 17095944

55 Thuzar M, Malabu UH, Tisdell B, Sangla KS. Use of a standardised diabetic ketoacidosis management protocol improved clinical outcomes. Diabetes Res Clin Pract 2014;104:e8-11. 10.1016/i. diabres.2014.01.016 pmid:24507867.

56 Ullal J, Aloi JA, Reyes-Umpierrez D, et al. Comparison of ComputerGuided Versus Standard Insulin Infusion Regimens in Patients With Diabetic Ketoacidosis. J Diabetes Sci Technol 2018;12:39-46. 10.1177/1932296817750899 pmid:29291648

57 Laliberte B, Yeung SYA, Gonzales IP. Impact of diabetic ketoacidosis management in the medical intensive care unit after order set implementation. Int J Pharm Pract 2017;25:238-43. 10.1111 ijpp.12346 pmid:28338247.

58 Martin C, McKinney ZJ, Hoody D, Fish L. Diabetic ketoacidosis critical care pathway implementation: Incorporation into emr significantly decreases length of stay. Endocr Pract 2016;22:673-8. 10.4158/EP151073. OR pmid:27176140.

59 Devalia B. Adherance to protocol during the acute management of diabetic ketoacidosis: would specialist involvement lead to better outcomes?Int/ Clin Pract 2010;64:1580-2. 10.1111/j.17421241.2010.02348.x pmid:20846206.

60 Tran TTT, Pease A, Wood AJ, et al. Review of Evidence for Adult Diabetic Ketoacidosis Management Protocols. Front Endocrinol (Lausanne) 2017;8:106. 10.3389/fendo.2017.00106 pmid:28659865.

61 Patiño AM, Marsh RH, Nilles El, Baugh CW, Rouhani SA, Kayden S. Facing the Shortage of IV Fluids - A Hospital-Based Oral Rehydration Strategy. N EnglJ Med 2018;378:1475-7. 10.1056/ NEJMp1801772 pmid:29561701.

62 Misra S, Oliver NS. Utility of ketone measurement in the prevention, diagnosis and management of diabetic ketoacidosis. Diabet Med 2015;32:14-23.10.1111/dme.12604 pmid:25307274.

63 Dhatariya KK, Vellanki P. Treatment of Diabetic Ketoacidosis (DKA) / Hyperglycemic Hyperosmolar State (HHS): Novel Advances in the Management of Hyperglycemic Crises (UK Versus USA). Curr Diab Rep 2017; 17:33. 10.1007/s11892-017-0857-4 pmid:28364357.

64 Ginde AA, Pelletier AJ, Camargo CA Jr. National study of U.S. emergency department visits with diabetic ketoacidosis, 1993-2003. Diabetes Care 2006:29:2117-9. 10.2337/dc06-0627 pmid:16936163.

65 Sampson M, Jones C. Joint British Diabetes Societies (JBDS) for Inpatient Care. Joint British Diabetes Societies for Inpatient Care: clinical guidelines and improving inpatient diabetes care. Diabet Med 2018;35:988-91. 10.1111/dme.13672 pmid:30152584.

66 Krentz Al, Hale PI, Singh BM, Nattrass M. The effect of glucose and insulin infusion on the fall of ketone bodies during treatment of diabetic ketoacidosis. Diabet Med 1989;6:31-6. 10.1111/j.1464-5491.1989. tb01135.x pmid:2522371.

67 Karajgikar ND, Manroa P, Acharya R, et al. Addressing pitfalls in management of diabetic ketoacidosis (DKA) with a standardized protocol. Endocr Pract2019.10.4158/EP-2018-0398 pmid:30657360.

68 Haas NL, Gianchandani RY, Gunnerson KJ, et al. The Two-Bag Method for Treatment of Diabetic Ketoacidosis in Adults. J Emerg Med 2018;54:593 9.10.1016/j.jemermed.2018.01.004 pmid:29628184.

69 Munir I, Fargo R, Garrison R, et al. Comparison of a 'two-bag system' versus conventional treatment protocol ('one-bag system') in the management of diabetic ketoacidosis. BMJ Open Diabetes Res Care 2017;5:e000395. 10.1136/bmjdrc-2017-000395 pmid:28878933.

70 Mazer-Amirshahi M, Fox ER. Saline Shortages - Many Causes, No Simple Solution. N Engl / Med 2018;378:1472-4. 10.1056/ NEJMp1800347 pmid.29561694.

71 Kitabchi AE, Murphy MB, Spencer J, Matteri R, Karas J. Is a priming dose of insulin necessary in a low-dose insulin protocol for the treatment of diabetic ketoacidosis?Diabetes Care 2008;31:2081-5. 10.2337/dc080509 pmid:18694978.

72 Cardoso L, Vicente N, Rodrigues D, Gomes L, Carrilho F. Controversies in the management of hyperglycaemic emergencies in adults with diabetes. Metabolism 2017;68:43-54.10.1016/j. metabol.2016.11.010 pmid:28183452

73 Loh TP, Saw S, Sethi SK. Bedside monitoring of blood ketone for management of diabetic ketoacidosis: proceed with care DiabetMed 2012:29.827-8,10.1111/j.14645491.2011.03490.x pmid:21999575.

74 Hsia E, Seggelke S, Gibbs J, et al. Subcutaneous administration of glargine to diabetic patients receiving insulin infusion prevents rebound hyperglycemia. J Clin Endocrinol Metab 2012;97:3132-7. 10.1210/ ic. 2012-1244 pmid:22685233.
75 Doshi P, Potter AJ, De Los Santos D, Banuelos R, Darger BF, Chathampally Y Prospective randomized trial of insulin glargine in acute management of diabetic ketoacidosis in the emergency department: a pilot study. Acad Emerg Med 2015;22:657-62.10.1111/ acem.12673 pmid:26013711.

76 Andrade-Castellanos CA, Colunga-Lozano LE, Delgado-Figueroa N, GonzalezPadilla DA. Subcutaneous rapid-acting insulin analogues for diabetic ketoacidosis. Cochrane Database Syst Rev 2016;(1):CD011281.pm id:26798030.

77 Ersöz HO, Ukinc K, Köse M, et al. Subcutaneous lispro and intravenous regular insulin treatments are equally effective and safe for the treatment of mild and moderate diabetic ketoacidosis in adult patients. Int/Clin Pract 2006:60:429-33.10.1111/j.13685031.2006.00786.x pmid:16620355.

78 Umpierrez GE, Cuervo R, Karabell A, Latif K, Freire AX, Kitabchi AE. Treatment of diabetic ketoacidosis with subcutaneous insulin aspart. Diabetes Care 2004;27:1873-8. 10.2337/diacare.27.8.1873 pmid:15277410.

79 Umpierrez GE, LatifK, Stoever ], et al. Efficacy of subcutaneous insulin lispro versus continuous intravenous regular insulin for the treatment of patients with diabetic ketoacidosis. Am J Med 2004;117:291-6. 10.1016/j. amjmed.2004.05.010 pmid:15336577.

80 Karoli R, Fatima J, Salman T, Sandhu S, Shankar R. Managing diabetic ketoacidosis in non-intensive care unit setting: Role of insulin analogs. Indian I Pharmacol 2011:43:398-401.10.4103/0253 7613.83109 pmid:21844993.

81 Chua HR, Schneider A, Bellomo R. Bicarbonate in diabetic ketoacidosis - a systematic review. Ann Intensive Care 2011;1:23. 10.1186/2110-5820-1 23 pmid:21906367.

82 Frontino G, Bonfanti R, Rigamonti A, et al. Management of hyperosmolar hyperglycaemic state in adults with diabetes. DiabetMed 2016;33:552. 10.1111/dme.12866 pmid:26206227

83 Magaji V, NayakS, Donihi AC, et al. Comparison of insulin infusion protocols targeting 110-140 mg/dL in patients after cardiac surgery. Diabetes Techno Ther 2012;14:1013-7. 10.1089/dia.2012.0114 pmid:23046399.

84 George S, Dale J, Stanisstreet D. Joint British Diabetes Societies (JBDS) for Inpatient Care JBDS Medical VRIII Writing Group. A guideline for the use of variable rate intravenous insulin infusion in medical inpatients. DiabetMed 2015;32:706-13.10.1111/dme.12756 pmid:25980646.

85 Mays JA, Jackson KL. Derby TA, etal. An Evaluation of Recurrent Diabetic Ketoacidosis, Fragmentation of Care, and Mortality Across Chicago, Illinois. Diabetes Care 2016;39:1671-6. 10.2337/dc16-0668 pmid:27422579.

86 Donihi AC. Practical Recommendations for Transitioning Patients with Type 2 Diabetes from Hospital to Home. Curr Diab Rep 2017;17:52. 10.1007/ s11892-017-0876-1 pmid:28573408

87 Vellanki P, Umpierrez GE. Increasing Hospitalizations for DKA: A Need for Prevention Programs. Diabetes Care 2018;41:1839-41. 10.2337/dci18 0004 pmid:30135197.

88 Kimmel B, Sullivan MM, Rushakoff RJ. Survey on transition from inpatient to outpatient for patients on insulin: what really goes on at home? Endocr Pract 2010;16:785-91.10.4158/EP10013.0R pmid:20350914.

89 Krall JS, Donihi AC, Hatam M, KoshinskyJ, Siminerio L. The Nurse Education and Transition (NEAT) model: educating the hospitalized patient with diabetes. Clin Diabetes Endocrinol 2016;2:1. 10.1186/s40842-016 0020-1 pmid:28702237.

90 Evans NR, Richardson LS, Dhatariya KK, Sampson MI. Diabetes specialist nurse telemedicine: admissions avoidance, costs and casemix. Eur Diabetes Nurs 2012;9:17-2110.1002/edn.198.

91 Berg EG. Sick-day rules: What to do when you are feeling under the weather. Diabetes Forecast 2013;66:32-3.pmid:24224354.

92 Auchterlonie A, Okosieme OE. Preventing diabetic ketoacidosis: do patients adhere to sick-day rules?Clin Med (Lond) 2013:13:120. 10.7861/ clinmedicine.13-1-120 pmid:23472520.

93 Scheen AJ. Pharmacokinetic Characteristics and Clinical Efficacy of an SGLT2 Inhibitor Plus DPP-4 Inhibitor Combination Therapy in Type 2 Diabetes. Clin Pharmacokinet 2017;56:703-18. 10.1007/s40262-016-04989 pmid:28039605.

94 Rosenbloom AL. Intracerebral crises during treatment of diabetic ketoacidosis. Diabetes Care 1990;13:22-33.10.2337/ diacare.13.1.22 pmid:2105195

95 Kuppermann N, Ghetti S, Schunk JE, et al. PECARN DKA FLUID Study Group. Clinical Trial of Fluid Infusion Rates for Pediatric Diabetic Ketoacidosis. N Engl JMed 2018:378:2275-87.10.1056/NEIMoa1716816 pmid:29897851.

96 Wolfsdorf JI, Glaser N, Agus M, et al. ISPAD Clinical Practice Consensus Guidelines 2018: Diabetic ketoacidosis and the hyperglycemic hyperosmolar state. Pediatr Diabetes 2018;19(Suppl 27):155-77. 10.1111/pedi.12701 pmid:29900641.

97 Mårtensson।, Bailey M, Venkatesh B, et al. Intensity of early correction of hyperglycaemia and outcome of critically ill patients with diabetic ketoacidosis. Crit Care Resusc 2017;19:266-73.pmid:28866977.

98 Dhatariya KK. Diabetic ketoacidosis. BMJ 2007;334:1284-5. 10.1136/ bmj.39237.661111.80 pmid:17585123.

99 Van Zyl DG, Rheeder P, Delport E. Fluid management in diabetic-acidosis-Ringer's lactate versus normal saline: a randomized controlled trial OIM 2012;105:337-43.10.1093/qjmed/hcr226 pmid:22109683.

100 Mahler SA, Conrad SA, Wang H, Arnold TC. Resuscitation with balanced electrolyte solution prevents hyperchloremic metabolic acidosis in patients with diabetic ketoacidosis. Am J Emerg Med 2011;29:670-4. 10.1016/j.ajem.2010.02.004 pmid:20825879. 
101 Issa M, Alqahtani F, Ziada KM, et al. Incidence and Outcomes of Non-ST Elevation Myocardial Infarction in Patients Hospitalized with Decompensated Diabetes. Am J Cardiol 2018;122:1297-302. 10.1016/j.amjcard.2018.07.004 pmid:30131108

102 Chen YL, Weng SF, Yang CY, Wang JJ, Tien KJ. Long-term risk of stroke in type 2 diabetes patients with diabetic ketoacidosis: A population-based, propensity score-matched, longitudinal follow-up study. Diabetes Metab 2017;43:223-8. 10.1016/. diabet.2016.11.003 pmid:28129999.

103 Azevedo LC, Choi H, Simmonds K, Davidow J, Bagshaw SM. Incidence and long-term outcomes of critically ill adult patients with moderate-tosevere diabetic ketoacidosis: retrospective matched cohort study. Crit Care 2014:29:971-7.10.1016//. jcrc.2014.07.034 pmid:25220529.
104 Bradford AL, Crider CC, Xu X, Naqvi SH. Predictors of Recurrent Hospital Admission for Patients Presenting With Diabetic Ketoacidosis and Hyperglycemic Hyperosmolar State. J Clin Med Res 2017;9:35-9. 10.14740/jocmr2792w pmid:27924173.

105 Gibb FW, Teoh WL, Graham J, Lockman KA. Risk of death following admission to a UK hospital with diabetic ketoacidosis. Diabetologia 2016:59:2082-7. 10.1007/s00125-016-4034-0 pmid:27397023.

106 Isidro ML, Jorge S. Recreational drug abuse in patients hospitalized for diabetic ketosis or diabetic ketoacidosis. Acta Diabetol 2013;50:183-7. 10.1007/s00592-010-0243-z pmid:21136122.

107 McCoy RG, Herrin J, Lipska KJ, Shah ND. Recurrent hospitalizations for severe hypoglycemia and hyperglycemia among U.S. adults with diabetes. J Diabetes Complications 2018;32:693-701. 10.1016/j. jdiacomp.2018.04.007 pmid:29751961. 